\title{
INVESTIGATIONS ON THE POSSIBLE BENEFICIAL EFFECTS OF A CENTERED ORBIT ON THE PERFORMANCE OF THE \\ LOW BETA-Y LATTICE
}

The submitid manuscripe has been authored by 3 contracitor of the U.S. Govarnment under consrisct No. W.31.109ENG.38. Accordingly. the U. S. Government retains a nonexclusive, royalty-free license to oublish or reproduce the published form of this contribution, or sllow ochers to do so, to U. S. Government purposes.

\author{
Annick Ropert, ESRF
}

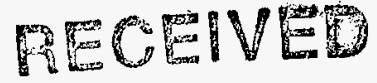

MAR 301998

\section{Motivation}

The APS storage ring is routinely operated with beam steering for the users. The resulting orbit distortions (local bumps at the source point locations might reach 1 to $2 \mathrm{~mm}$ ) could have a detrimental impact on the lattice performance via the off-centered path of the beam in the sextupoles. Resulting effects could include the following: modulation of the lattice functions, emittance growth, dynamic aperture and energy acceptance reduction, and lifetime reduction.

The goal of the present study was to make a comparative analysis of the performance of the low beta-y optics when the machine is operated with the user's orbit and with a centered orbit. Theoretical investigations as well as results of machine studies performed during the October November run are presented in this Note. Since, to some extend, gradient errors could also induce similar perturbations, the unambiguous identification of the source of errors might be difficult. Some possibilities for discriminating between the different errors are also discussed.

\section{Basic features of the low beta-y lattice}

In order to accommodate smaller-gap vacuum vessels, a new version of the lattice with $\beta_{y}=3 \mathrm{~m}$ instead of $10 \mathrm{~m}$ in the straight sections is under testing. While keeping the horizontal tune at the same value $\left(v_{x}=35.2\right)$, several lattice configurations can be optimized by leaving the achromat quadrupoles unchanged and adjusting the triplet in the straight section in order to match the required constraints. Figure 1 shows the evolution of lattice functions for the low $\beta_{y}$ lattice tuned at $v_{y}=19.3$ as compared to those of the standard optics. The emittance provided by this tuning is $\varepsilon_{\mathrm{x}}=7.6 \mathrm{~nm}$. (Although the quadrupole settings in the achromat remain identical, the slight changes in the $\beta_{x}$ function and in the dispersion at the entrance of the dipole result in an emittance reduction with respect to the standard 8.3-nm figure.) As illustrated in Figure 2, lower emittances could be achieved by tuning the machine on other vertical tunes, but these settings present some drawbacks: large vertical $\beta$ functions $\left(v_{y}=16.3\right)$ leading to a smaller vertical acceptance or tuning in the vicinity of a nonlinear coupling resonance $\left(v_{y}=17.3\right.$ and $v_{y}$ =18.3), thus reducing the dynamic aperture.

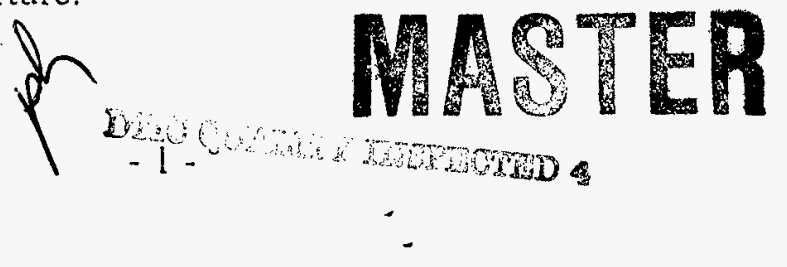




\section{DISCLAIMER}

This report was prepared as an account of work sponsored by an agency of the United States Government. Neither the United States Government nor any agency thereof, nor any of their employees, makes any warranty, express or implied, or assumes any legal liability or responsibility for the accuracy, completeness, or usefulness of any information, apparatus, product, or process disclosed, or represents that its use would not infringe privately owned rights. Reference herein to any specific commercial product, process, or service by trade name, trademark, manufacturer, or otherwise does not necessarily constitute or imply its endorsement, recommendation, or favoring by the United States Government or any agency thereof. The views and opinions of authors expressed herein do not necessarily state or reflect those of the United States Government or.any agency thereof. 


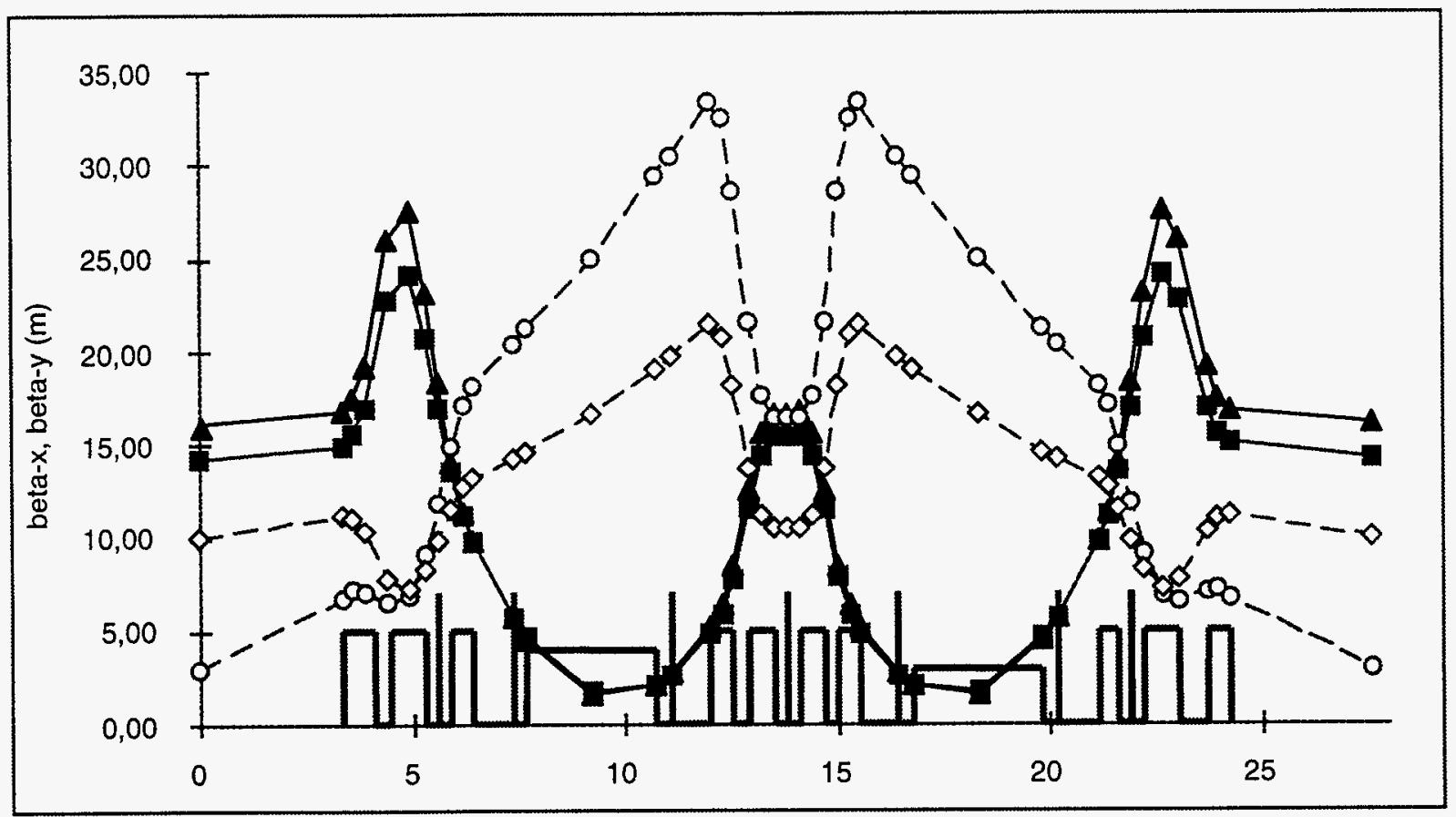

Figure 1. Lattice functions of the $v_{\mathrm{y}}=14.3$ and $v_{\mathrm{y}}=19.3$ optics

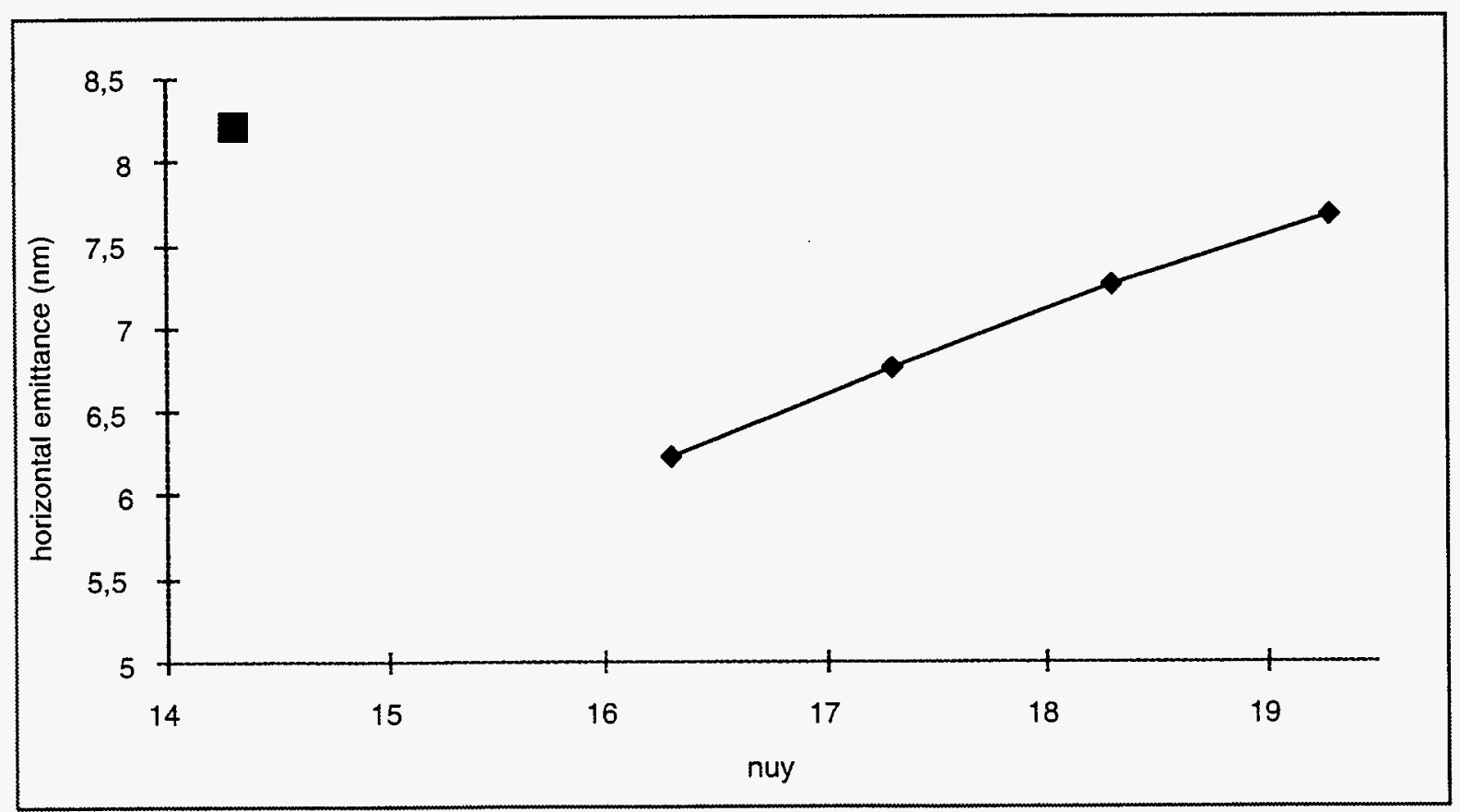

Figure 2. Emittance evolution of the different $v_{y}$ optics

The dynamic aperture of the ideal machine (no errors, no vacuum vessel)) is plotted in Figure 3 for on- and off-momentum particles $(\Delta \mathrm{p} / \mathrm{p}= \pm 2 \%)$. Chromaticities are set at $\xi_{\mathrm{x}}=1.5$ and $\xi_{\mathrm{y}}=4$. Harmonic sextupoles are set at $80 \mathrm{~A}\left(\mathrm{Hl}=1.165 \mathrm{~m}^{-2}\right)$ and $130 \mathrm{~A}\left(\mathrm{Hl}=-1.893 \mathrm{~m}^{-2}\right)$, 
respectively. The contour exhibits a strong dissymmetry for all momentum deviations. The evolution of the dynamic aperture induced by a scan of the harmonic sextupoles is shown in Figure 4. Apart from one set of sextupoles that leads to a significant reduction in the vertical plane, no significant effect can be seen.

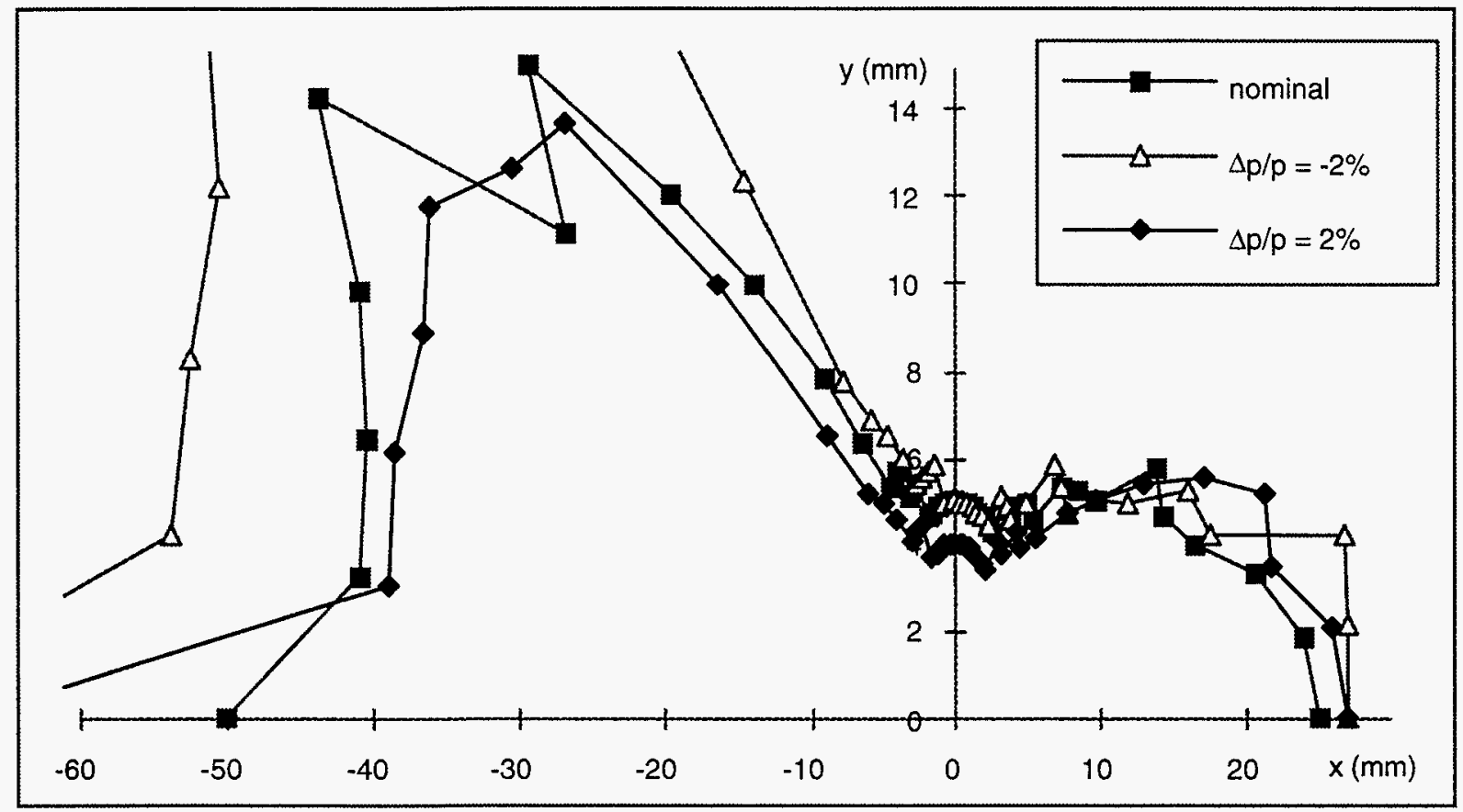

Figure 3. Dynamic aperture of the ideal machine

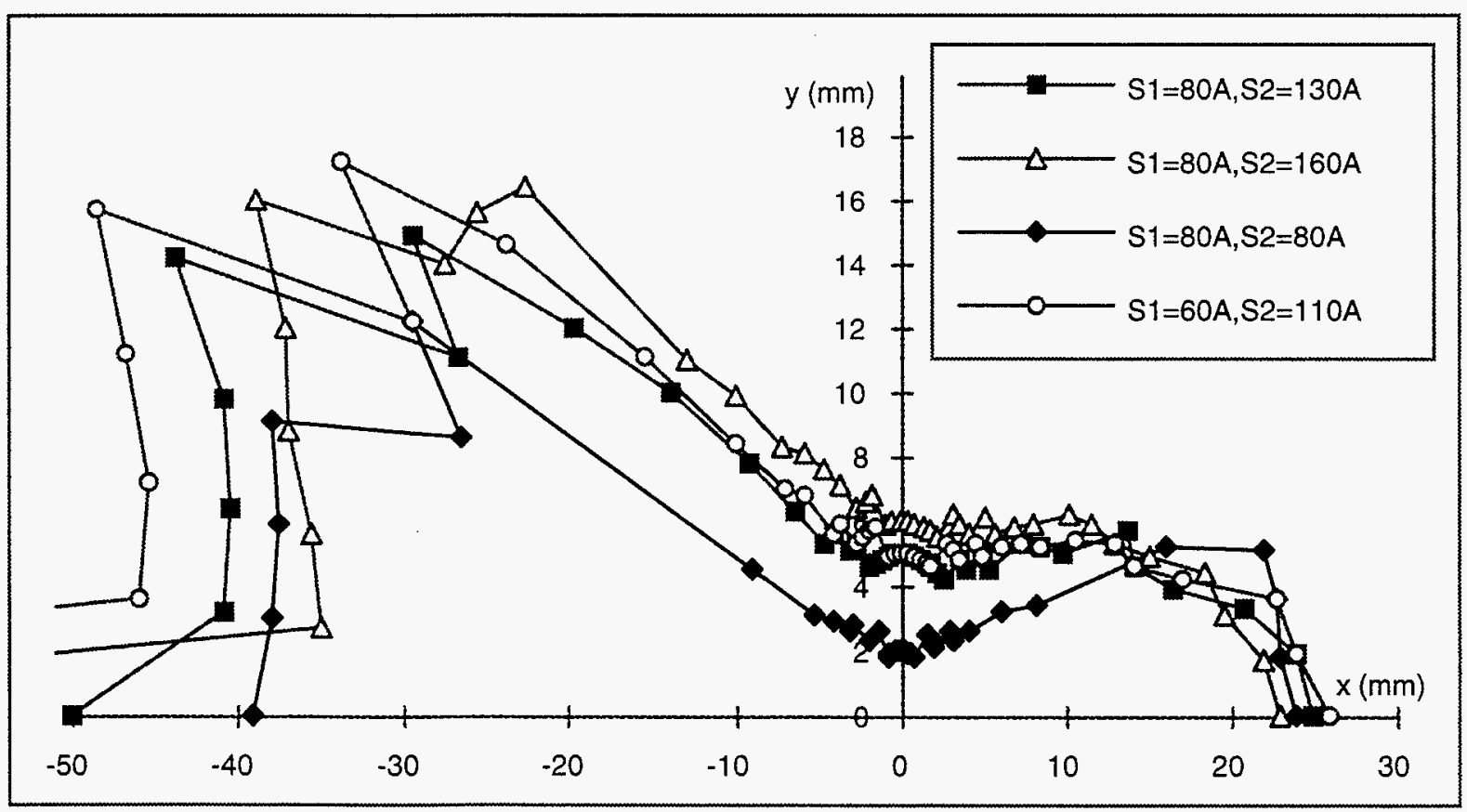

Figure 4. Effect of harmonic sextupoles on the dynamic aperture 
When an elliptical $( \pm 25 \mathrm{~mm} * \pm 4 \mathrm{~mm})$ vessel is considered, the effective dynamic aperture shrinks down, due to the horizontal-vertical coupling, as indicated in Figure 5. Present harmonic sextupoles induce very large tune shifts with amplitudes (see Figure 6). Whether new settings of these sextupoles could minimize these nonlinear effects and restore a dynamic aperture larger than the physical aperture has to be assessed.

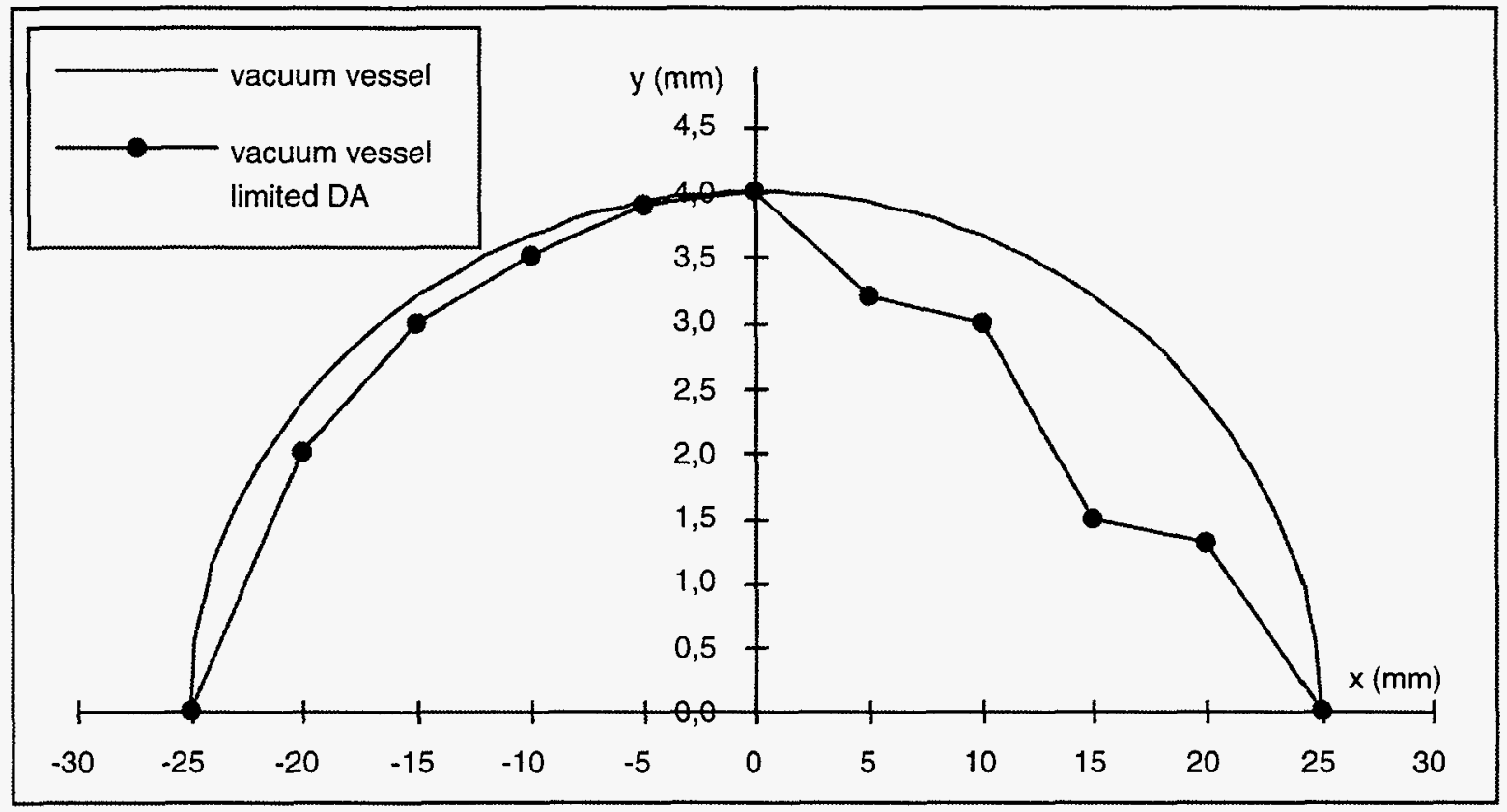

Figure 5. Effective dynamic aperture

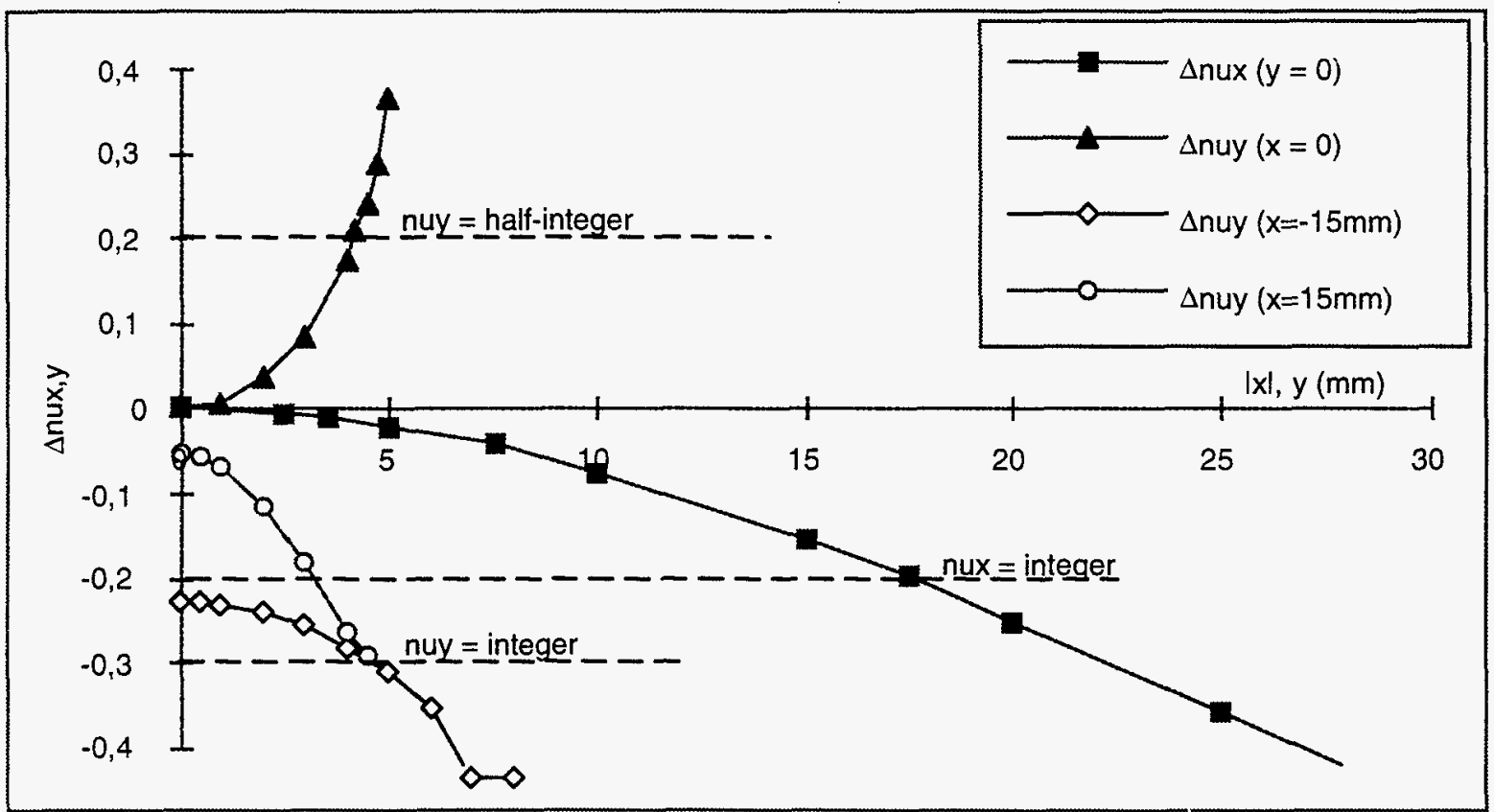

Figure 6. Tune shifts with amplitude 


\section{Effects of errors in the machine}

\section{Closed-orbit errors}

\subsection{Simulations}

The effects of closed-orbit errors have been simulated by using quadrupole alignment errors. Although the pattern of the resulting closed-orbit distortions is not identical to that of the real machine, the equivalent magnitudes of the rms distortions should qualitatively produce similar effects. Simulations were performed with ten different sets of errors (in order to get statistically valid results), inducing rms orbits of $0.25 \mathrm{~mm}$ and $0.5 \mathrm{~mm}$ in both planes. The main outcomes of the tracking simulations are as follows:

- Almost no tune changes $(<0.01)$ induced by the off-centered orbits.

- $\beta$-function modulation of a few \% in both planes.

- Modulation of the horizontal dispersion with a 5-fold periodicity resulting from the beating between the ring periodicity (40) and the betatron frequency $\left(v_{x}=35\right)$ as shown in Figure 7 for an rms orbit of $0.25 \mathrm{~mm}$. The amplitude of the modulation obviously increases with the closed orbit distortions.

- Strong reduction of the dynamic aperture (no vacuum vessel). The increased deterioration with increased closed-orbit distortions is illustrated in Figures 8 and 9.

- Significant blow-up of the horizontal emittance (Figure 10) and of the vertical emittance.

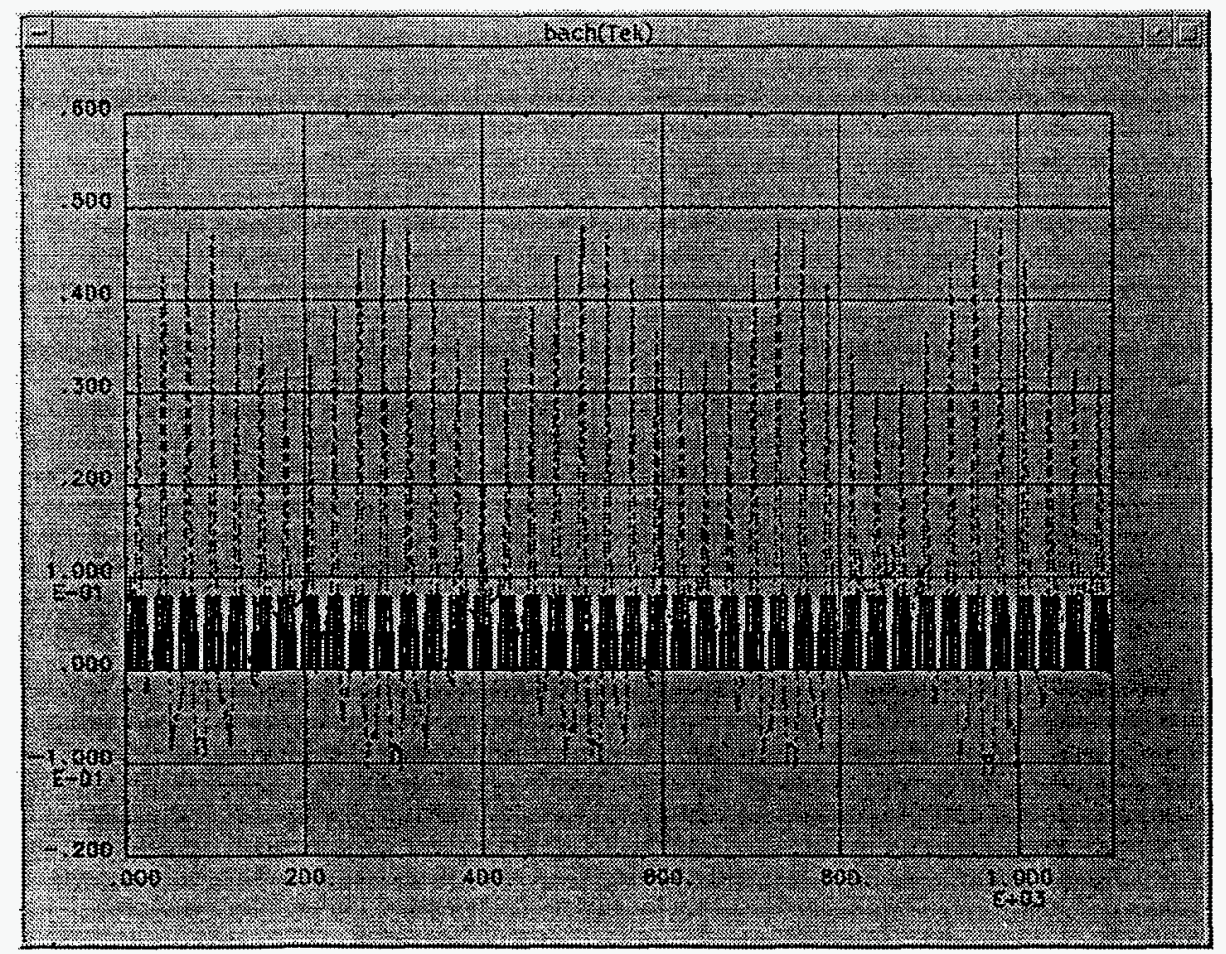

Figure 7. Horizontal dispersion with closed orbit errors 


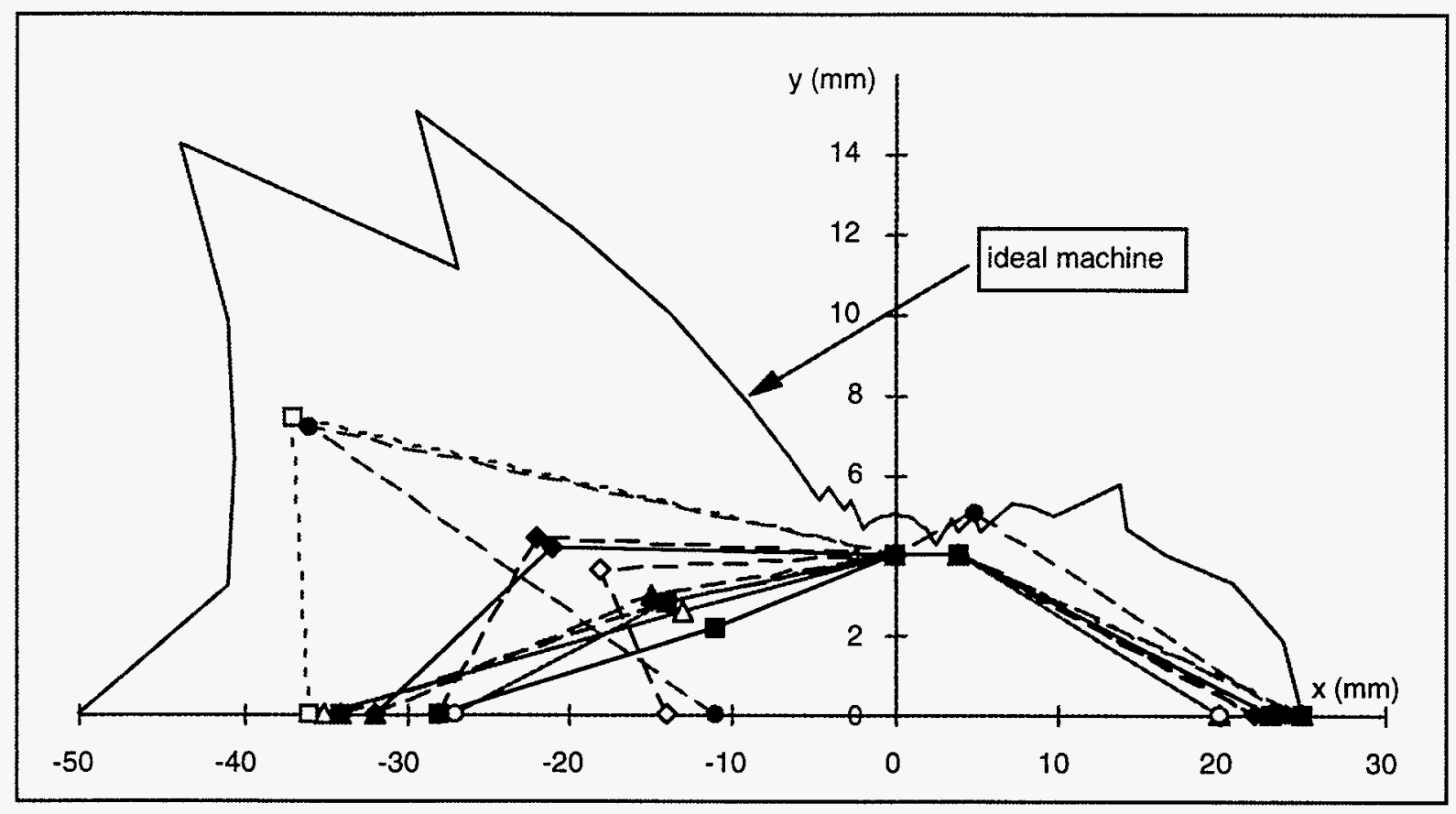

Figure 8. Dynamic aperture with $0.25-\mathrm{mm} \mathrm{rms}$ closed orbit

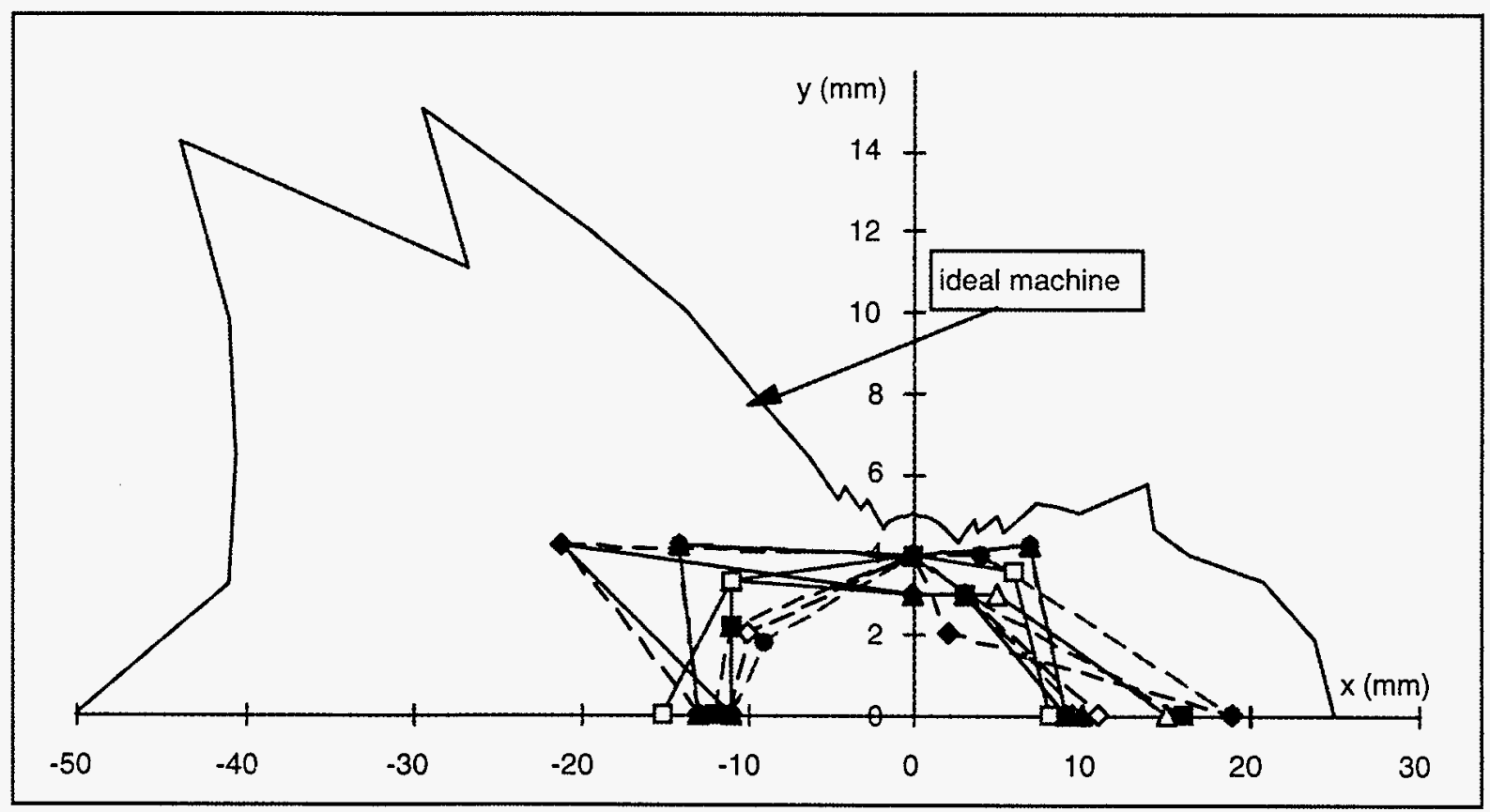

Figure 9 . Dynamic aperture with $0.50-\mathrm{mm}$ rms closed orbit 


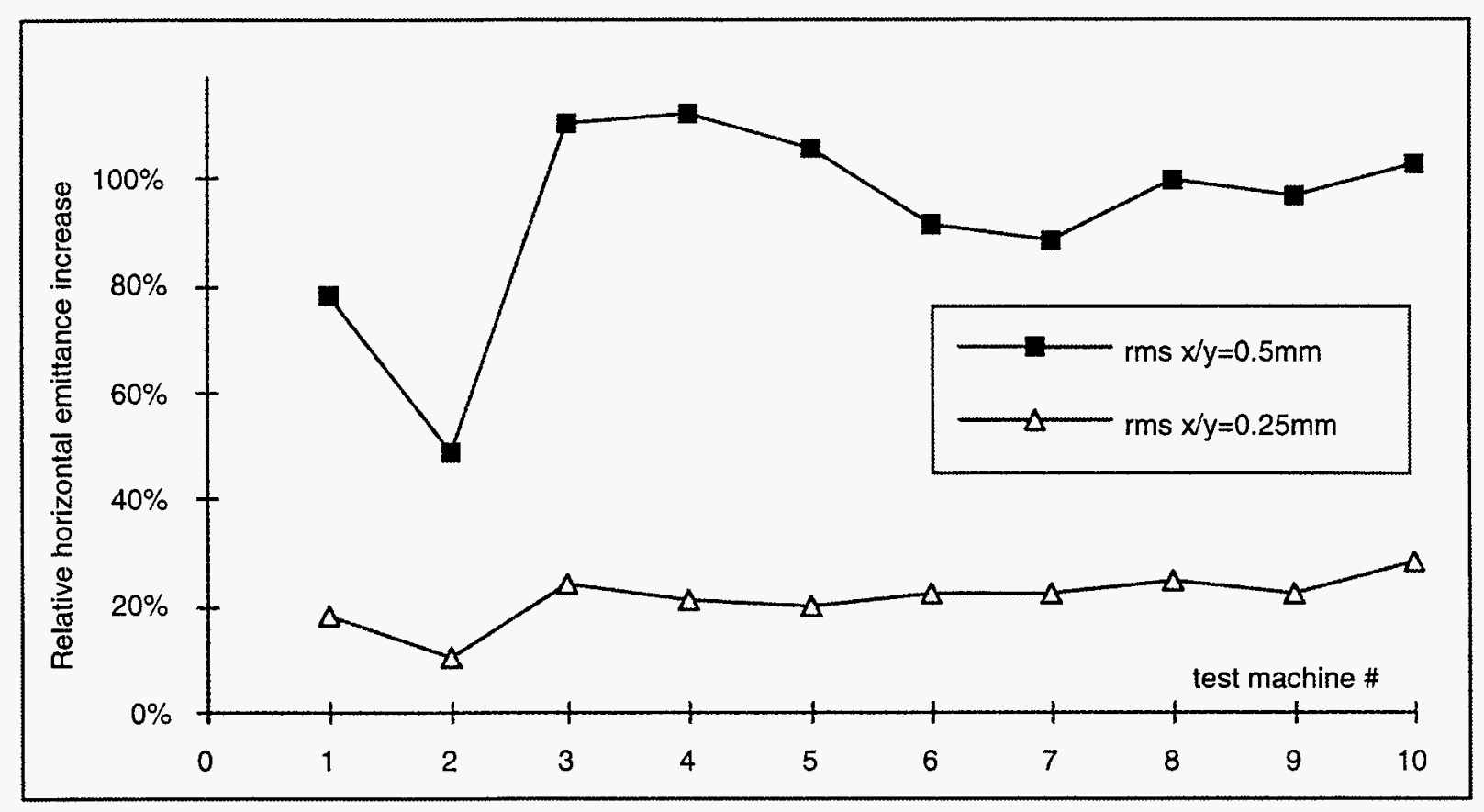

Figure 10. Horizontal emittance blow-up with closed-orbit distortions

\subsection{Experimental conditions}

Most of the tests were performed with the machine run at $10 \mathrm{~mA}$ with a group of six bunches or with a single 5-mA bunch. Three different types of orbit correction were used:

- User's orbit leading to rms values of the order of $0.5 \mathrm{~mm}$ in the horizontal/vertical plane.

- "Partially" centered orbit: rms values of the orbit remained large despite the fact that zero setpoints were used, global and fine corrections were made in both planes, and centering of P5 BPMs with the rf frequency was performed.

- Orbit centered at "best": with zero setpoints, we settled on the normal orbit correction for the horizontal plane that is used for the $v_{y}=14.3$ optics. The vertical plane used the P2 and P5 BPMs along with the V2 and BV4 correctors. Vertically, the orbit was well corrected at the P2s and P5s ( $<10$ microns). Horizontally, the value was more like $0.25 \mathrm{~mm}$ with an average orbit of $0.02 \mathrm{~mm}$.

\section{Gradient errors}

Given the values of the tunes in both planes that are close to 0.25 , little amplification of random gradient errors should occur. However, simulations for different sets of gradient errors with an rms value $\langle\Delta \mathrm{G} / \mathrm{G}\rangle=10^{-3}$ give the following results: 
- Noticeable tune shifts; with over 50 sets of errors, one gets:

$$
\begin{array}{ll}
\bar{v}_{x}=35.215 & \sigma_{v_{x}}=0.012 \\
\bar{v}_{y}=19.285 & \sigma_{v_{y}}=0.01
\end{array}
$$

- The modulation of the $\beta$-functions is somewhat larger than the one produced by 0.5 $\mathrm{mm}$ rms closed-orbit errors, as shown in Figure 11. It appears in both planes with equivalent amplitudes.

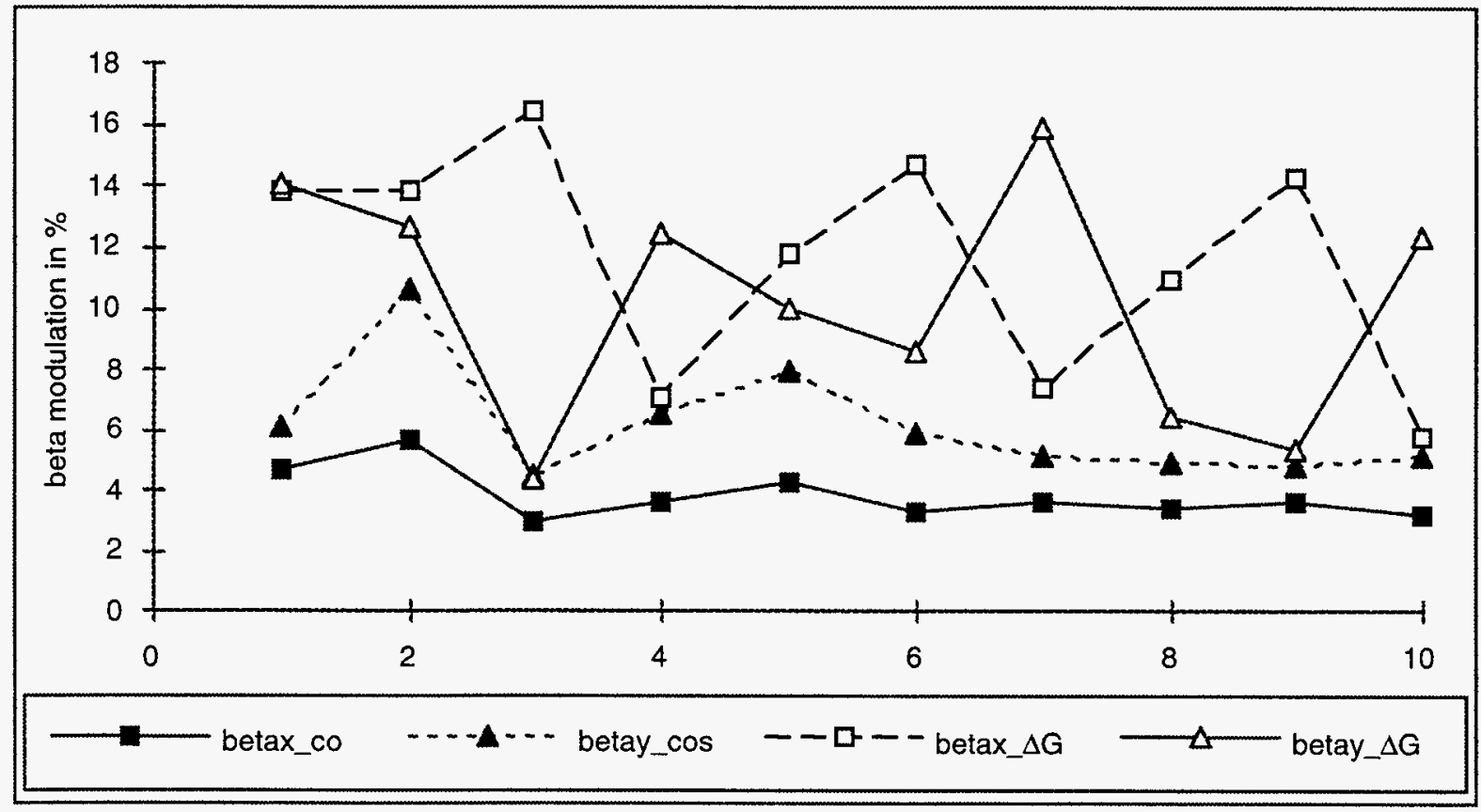

Figure 11. $\beta$-function modulation induced by errors

- The modulation of the horizontal dispersion (Figure 12) is very similar to that produced by closed-orbit errors.

It has to be pointed out that such a dispersion pattern could be produced by several means: as an example, Figure 13 shows the modulation induced by a $2 \%$ gradient error in a single Q5 quadrupole. In this particular example, other lattice characteristics are not significantly perturbed $(\Delta v \sim 0.01, \beta$-modulation of a few $\%)$.

- Almost no horizontal emittance blow-up (less than 3\%). 


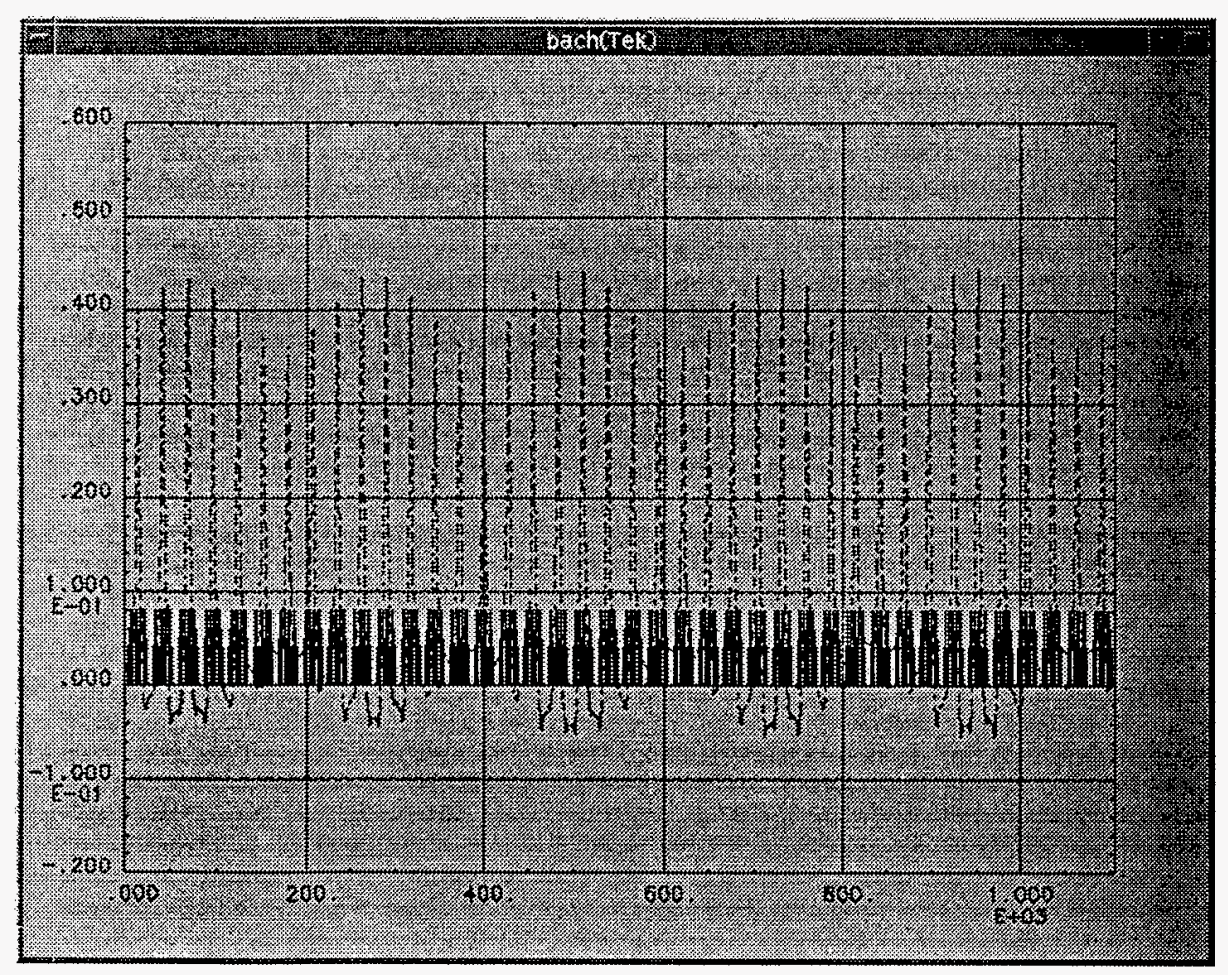

Figure 12. Horizontal dispersion with rms gradient errors

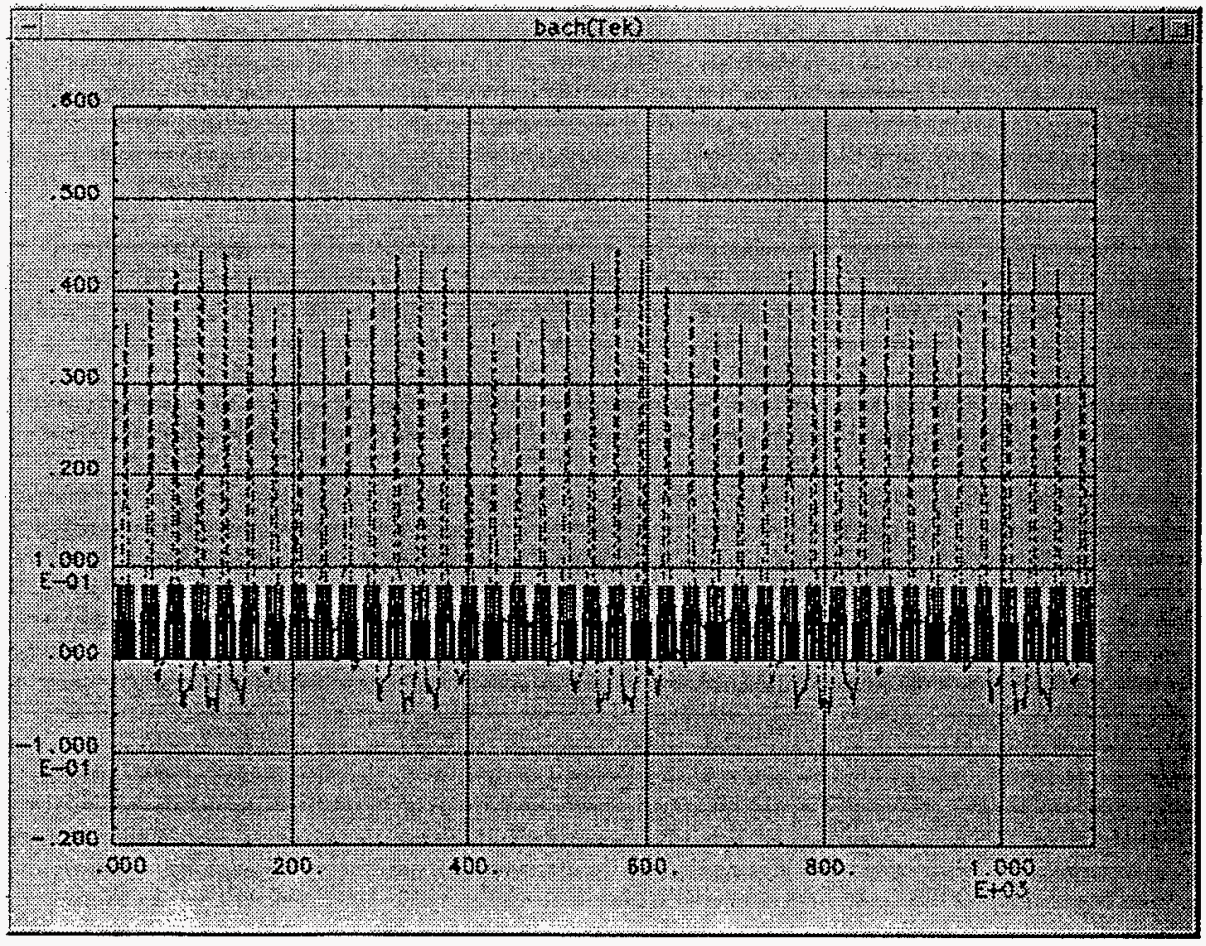

Figure 13. Horizontal dispersion with a single gradient error in a Q5 quadrupole 
In order to determine whether the experimentally observed distortions are more likely induced by quadrupole and/or power supplies calibration errors or generated by closed-orbit errors, the horizontal or vertical tune was moved towards the half-integer. Closed-orbit-related distortions should be minimized whilst the effects of focusing errors should be amplified.

\section{Energy acceptance}

The energy acceptance was measured, using two techniques:

- Perform an rf frequency scan by steps of $100 \mathrm{~Hz}$, starting at $\pm 500 \mathrm{~Hz}$ from the nominal of frequency and recording lifetime, betatron tunes with a $10 \mathrm{~mA}$ in 6 bunches beam. It must be mentioned that this experiment does not really represent the situation of Touschek-scattered particles since the whole beam is offset in energy. The energy acceptance is computed from the rf frequencies giving zero lifetime.

- Vary the rf voltage by steps of 0.5 MV and record the lifetime and synchrotron tune for a 10-mA beam in six bunches. As long as the energy acceptance is rf-related, the lifetime should increase with if voltage. Saturation occurs when the energy acceptance is limited by transverse considerations (dynamic or physical aperture). In terms of physical aperture, the injection septum is the limiting obstacle for particles that are Touschek scattered in the achromat and experience an induced betatron oscillation. For the low beta-y lattice, this limit corresponds to $\Delta \mathrm{p} / \mathrm{p}=4.5 \%$.

\section{1. rffrequency scan}

Measurements were performed on three occasions with different orbits. Chromaticities were measured at:

$$
\begin{array}{lll}
\xi_{\mathrm{x}}=0.38 \pm 0.17 & \xi_{\mathrm{y}}=4.18 \pm 0.12 & \text { (Oct19_partially centered orbit) } \\
\xi_{\mathrm{x}}=0.49 \pm 0.42 & \xi_{\mathrm{y}}=4.91 \pm 0.27 & \text { (Nov3_centered orbit) }
\end{array}
$$

The evolution of lifetime as a function of rf frequency is summarized in Figure 14. Whatever the orbit, there is a clear dissymmetry between the positive and negative energy sides. Surprisingly, the centered orbit corresponds to the smallest energy acceptance (see Table 1). 


\section{Table 1}

\begin{tabular}{c|c|c|c} 
& user's orbit & partially centered & centered \\
\hline$\Delta \mathrm{p} / \mathrm{p}$ & $-1.9 \% / 1.2 \%$ & $-2.2 \% / 1.7 \%$ & $-1.9 \% / 0.8 \%$
\end{tabular}

This is not understood yet. It can also be noticed that the lifetime significantly drops when the machine is run with centered orbits. This is most likely linked to smaller emittances, thus leading to a reduced Touschek lifetime.

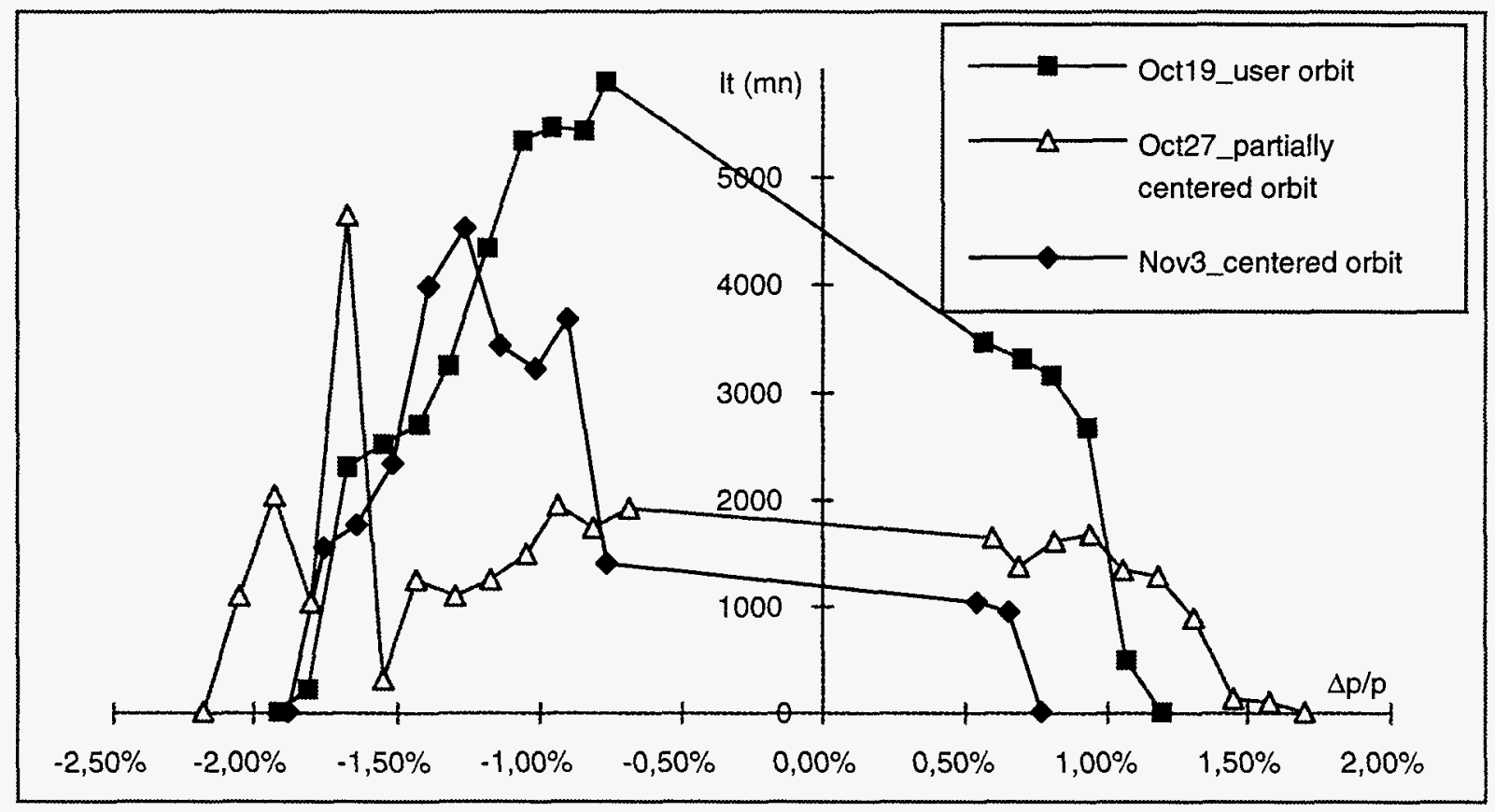

Figure 14. Lifetime evolution as a function of energy deviations

Figure 15 shows the comparison of measured and computed tunes versus energy deviations. Unfortunately, on the positive rf frequency side, the measured horizontal tune peak disappears. There is a very good agreement between simulations and experiments. The discrepancy appearing at large negative $\Delta p / p$ for the vertical tune comes from the difficulty in resolving the vertical peak on the network analyzer, as shown in Figure 16. 


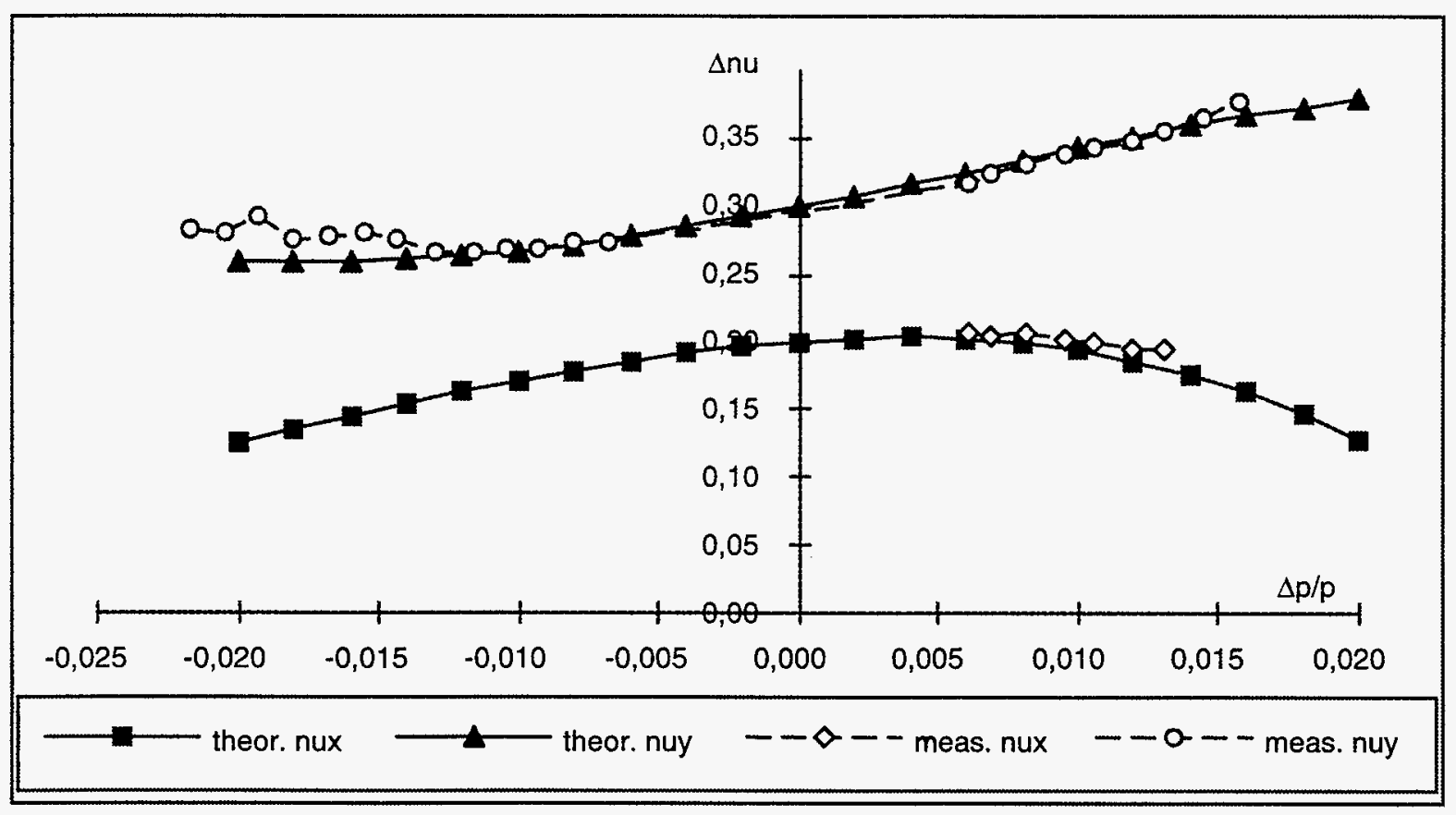

Figure 15. Tune dependence on energy deviations

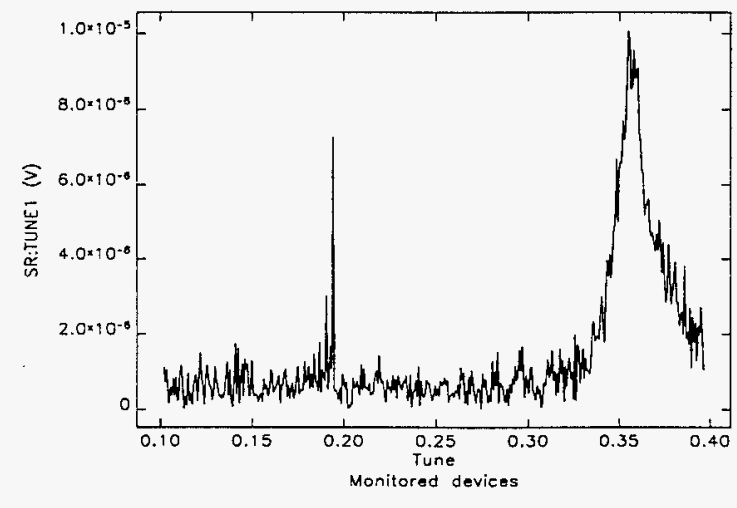

$\Delta f_{R F}=-1200 \mathrm{~Hz}$

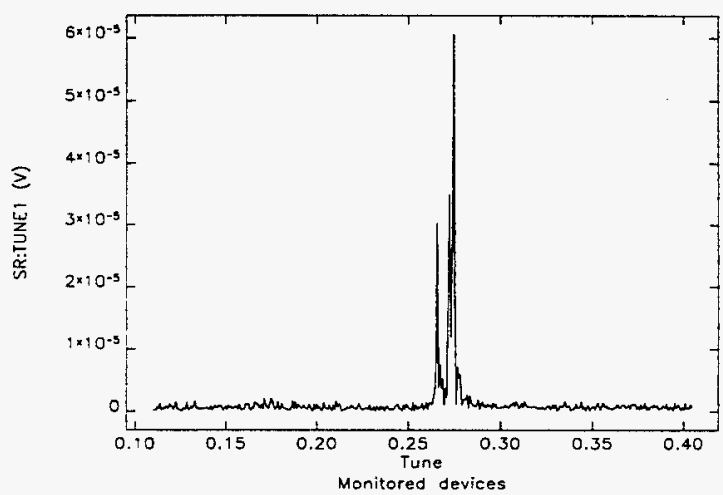

$\Delta f_{\mathrm{RF}}=1200 \mathrm{~Hz}$

Figure 16. Measured tunes

The tune excursions in the tune diagram for off-momentum particles are plotted in Figure 17. Apparently the systematic fifth-order resonance $4 v_{x}+v_{y}=160$ is not harmful. However its crossing might be the cause for the significant lifetime drop between $\Delta \mathrm{p} / \mathrm{p}= \pm 0.6 \%$ for the user's orbit lifetime curve. (A fine frequency scan of $\pm 500 \mathrm{~Hz}$ apart from the central frequency could be helpful to assess this assumption.) It seems that the limits of the energy acceptance are determined by the random third-order resonance $2 v_{x}-v_{y}=51$. A possible way of confirming this would be to remeasure the lifetime versus if frequency while moving the horizontal tune 
towards higher values. This would accordingly shift the tune path in the tune diagram and allow for larger $\Delta \mathrm{p} / \mathrm{p}$ excursions before crossing the resonance.

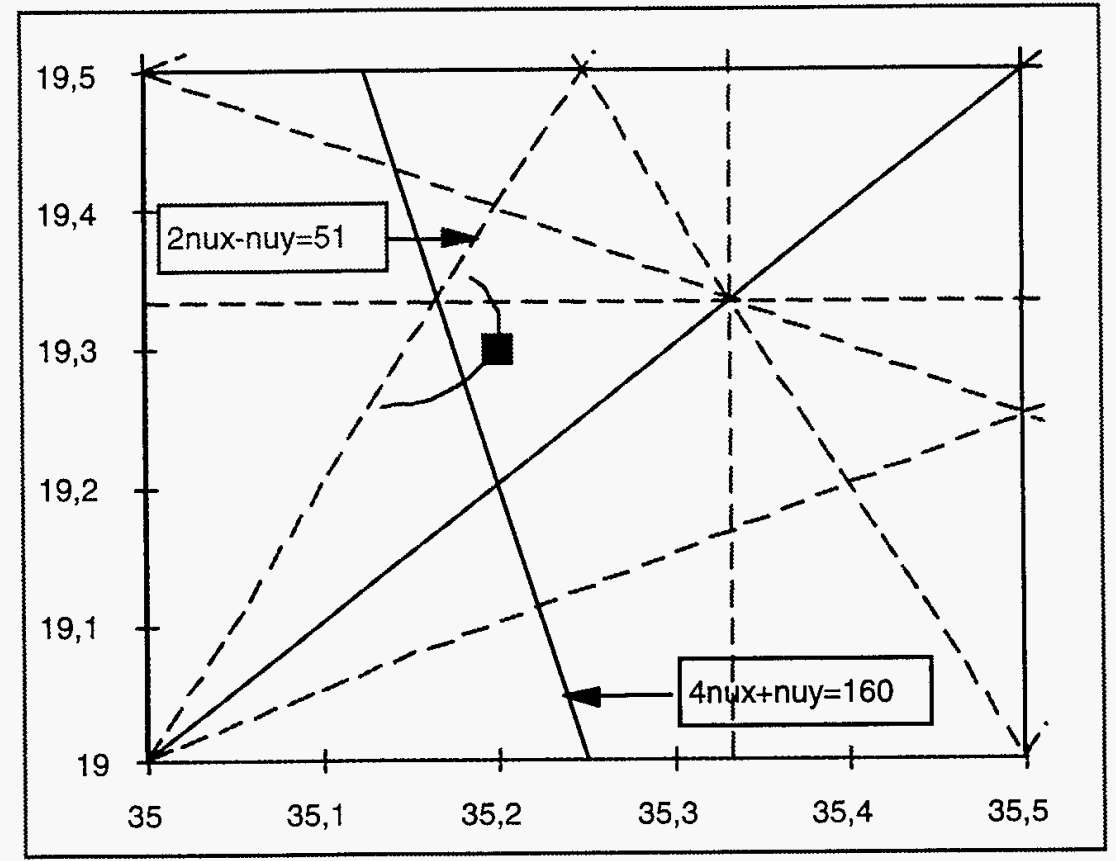

Figure 17. Tune paths for off-momentum particles

\section{If voltage scan}

Again measurements were carried out for a 10-mA beam in six bunches with the three different closed orbits. Figure 18 shows the lifetime as a function of the total gap rf voltage. No significant change of the point where the lifetime saturates can be observed (although the centered orbit curve has a tendency to saturate at lower rf voltage, which looks surprising given the fact that the machine should a priori be less perturbed than with a larger closed orbit).

The energy acceptance can be deduced by using the total gap voltage setpoint or the value deduced from the measurement of the synchrotron frequency when the lifetime starts to saturate. Using the $V_{\text {inferred }}$ value should eliminate possible miscalibration of the total gap voltage. On the other hand, the synchrotron frequency could be altered by the defocusing effect induced by the interaction of the single bunch with the vacuum pipe; it is expected that, with the actual charge per bunch $(1.66 \mathrm{~mA})$, this perturbation is negligible. Depending upon whether the rf voltage is taken from the direct measurement or is deduced from the measurement of the synchrotron frequency, the energy acceptance is $2.3 \%$ or $2.1 \%(2.1 \%$ or $1.9 \%$ for the centered orbit), i.e. smaller than the acceptance provided by the rf system ( $2.8 \%$ for $\left.V_{R F}=9.5 \mathrm{MV}\right)$. This clearly indicates that the reduced energy acceptance is induced by transverse limitations. 


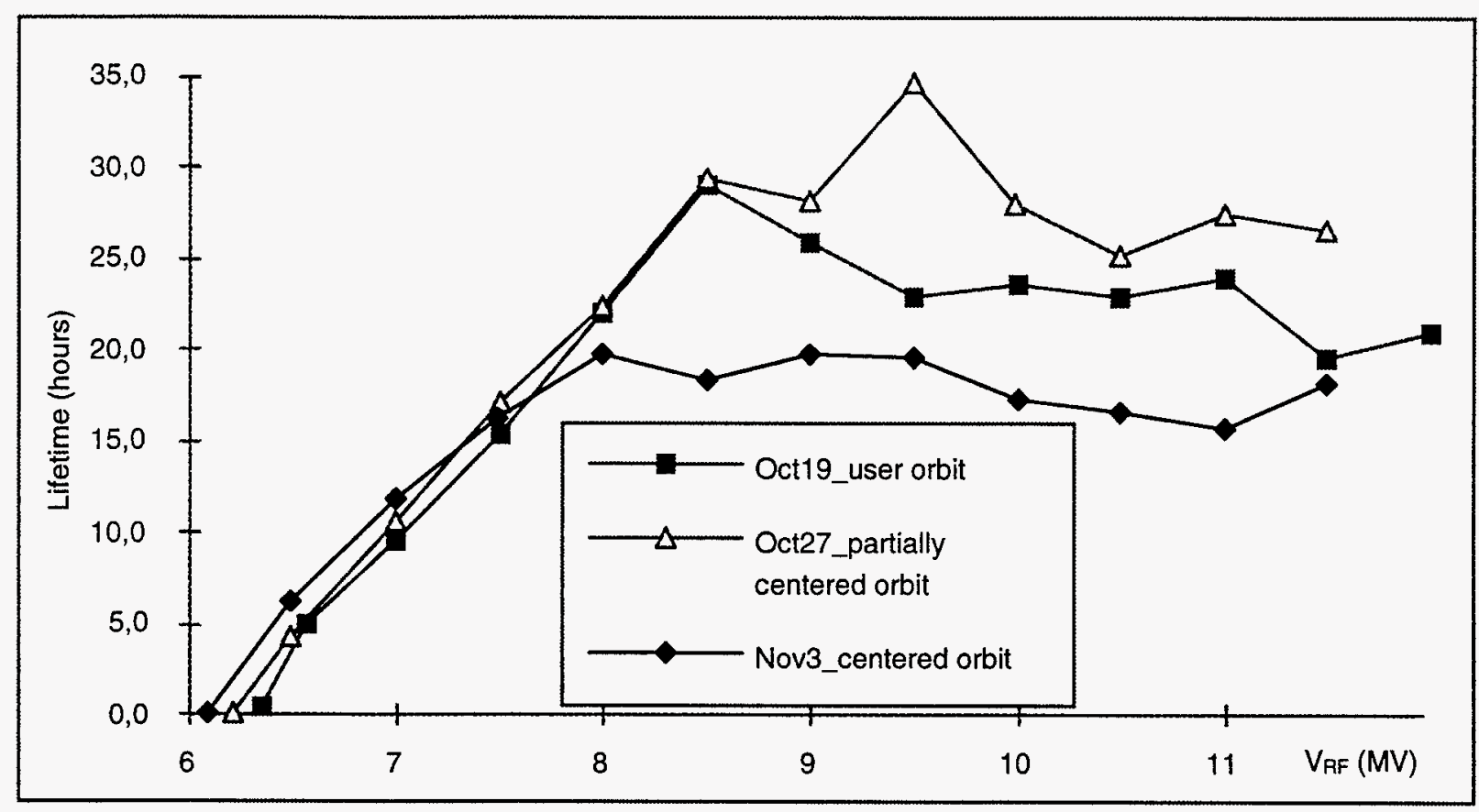

Figure 18. Lifetime versus total gap voltage

The comparison between the measured data (for the user's orbit) and the modeled ones is given in Figure 19. Theoretical emittances are used in the modeling $\left(\varepsilon_{\mathrm{x}}=7.6 \mathrm{~nm}, \sigma_{\mathrm{L}}=4.8 \mathrm{~mm}\right.$ for $\mathrm{V}_{\mathrm{RF}}=9.5 \mathrm{MV}$ ) and a $2 \%$ coupling is assumed. Beyond the point where the measured lifetime starts to saturate, a constant limiting $\Delta \mathrm{p} / \mathrm{p}$ of $2.09 \%$ is used in the simulations. The decreasing lifetime simply reflects the bunch shortening with increasing rf voltage. There is a relatively good matching between the computations and the measurements, although the slope of the two curves in the rf-limited zone has not exactly the same behavior. This agreement looks somewhat surprising, given the fact that some transverse emittance increase should be induced by the orbit distortions. In addition, bunch lengthening effects should also been considered. The $10 \%$ increase that has been used in Figure 19 does not dramatically change the matching. The measurements have to be reproduced while simultaneously recording the transverse emittances and the bunch lengths. 


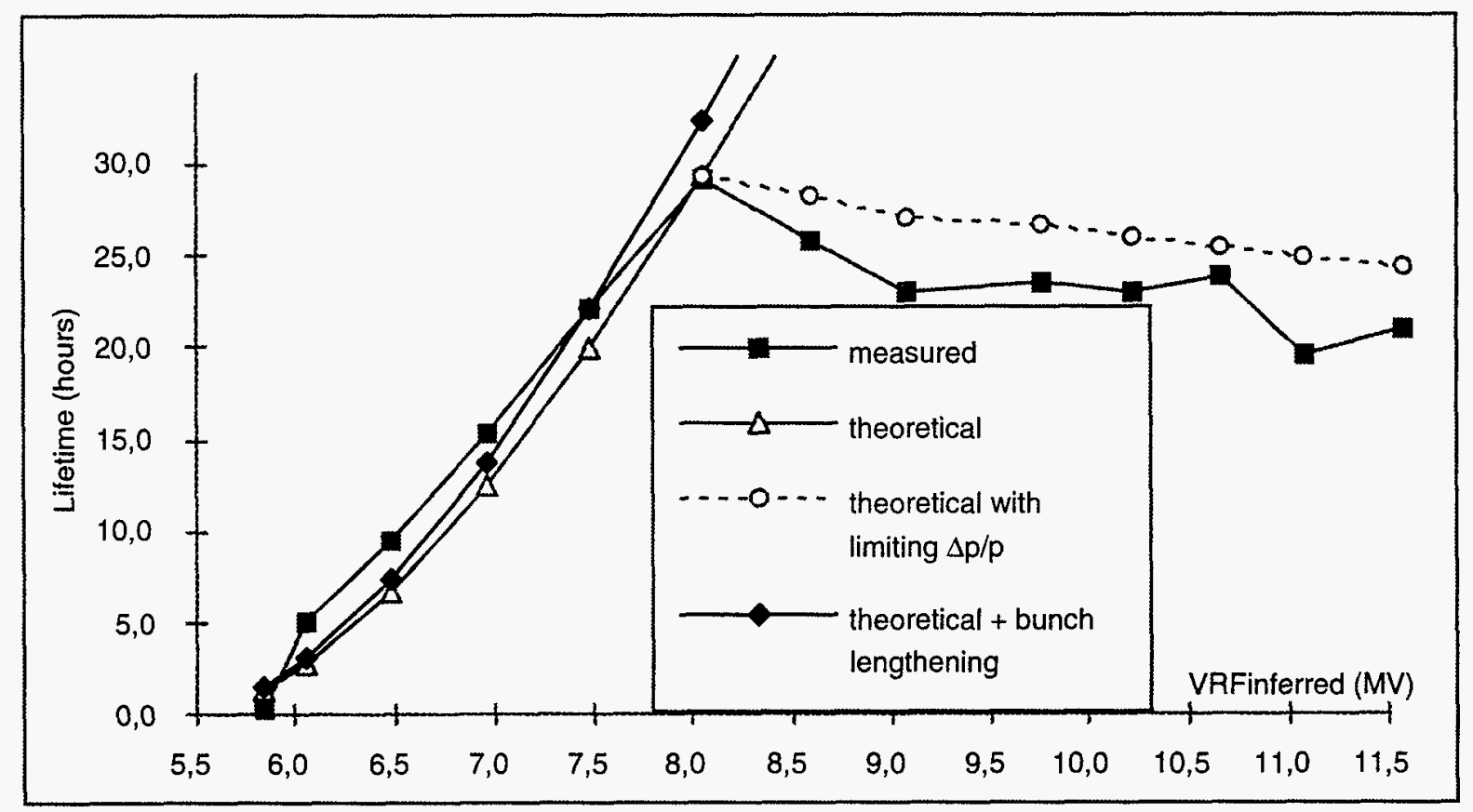

Figure 19. Comparison of measured and computed lifetimes versus rf voltage

\section{Dynamic aperture}

Measurements of the horizontal dynamic aperture were performed with a 5-mA single bunch in different orbit configurations by exciting a betatron oscillation of increasing amplitude with one of the injection kickers ( $\mathrm{K} 4$ was used) and recording intensity and BPM history (from S34A:P2 and S36A:P2). The betatron amplitude induced by the kicker is determined from the BPM history and scaled to a $\beta_{x}$ value of $16.03 \mathrm{~m}$ (theoretical value in the middle of the straight sections).

\section{Scan of harmonic sextupoles}

Figure 20 shows the processed results of harmonic sextupole scans ( $\mathrm{S} 1=80 \mathrm{~A}$ with $\mathrm{S} 2$ varying from 80 to $130 \mathrm{~A}, \mathrm{~S} 2=110 \mathrm{~A}$ with S1 varying from 60 to $100 \mathrm{~A}$ ), with nominal values of $\mathrm{S} 1=$ $80 \mathrm{~A}, \mathrm{~S} 2=130 \mathrm{~A}$. As expected from simulations (see Figure 4), the dependence on sextupole settings is very flat. The maximum stable amplitude is always smaller than $10 \mathrm{~mm}$ (Figure 21), which is far from the ideal dynamic aperture. This could possibly be due to a severe shrinkage of the dynamic aperture with errors (as sketched in Figures 8 and 9) or to a significant coupling of horizontal motion onto the vertical plane and a beam loss on small-gap ID vacuum vessels. 


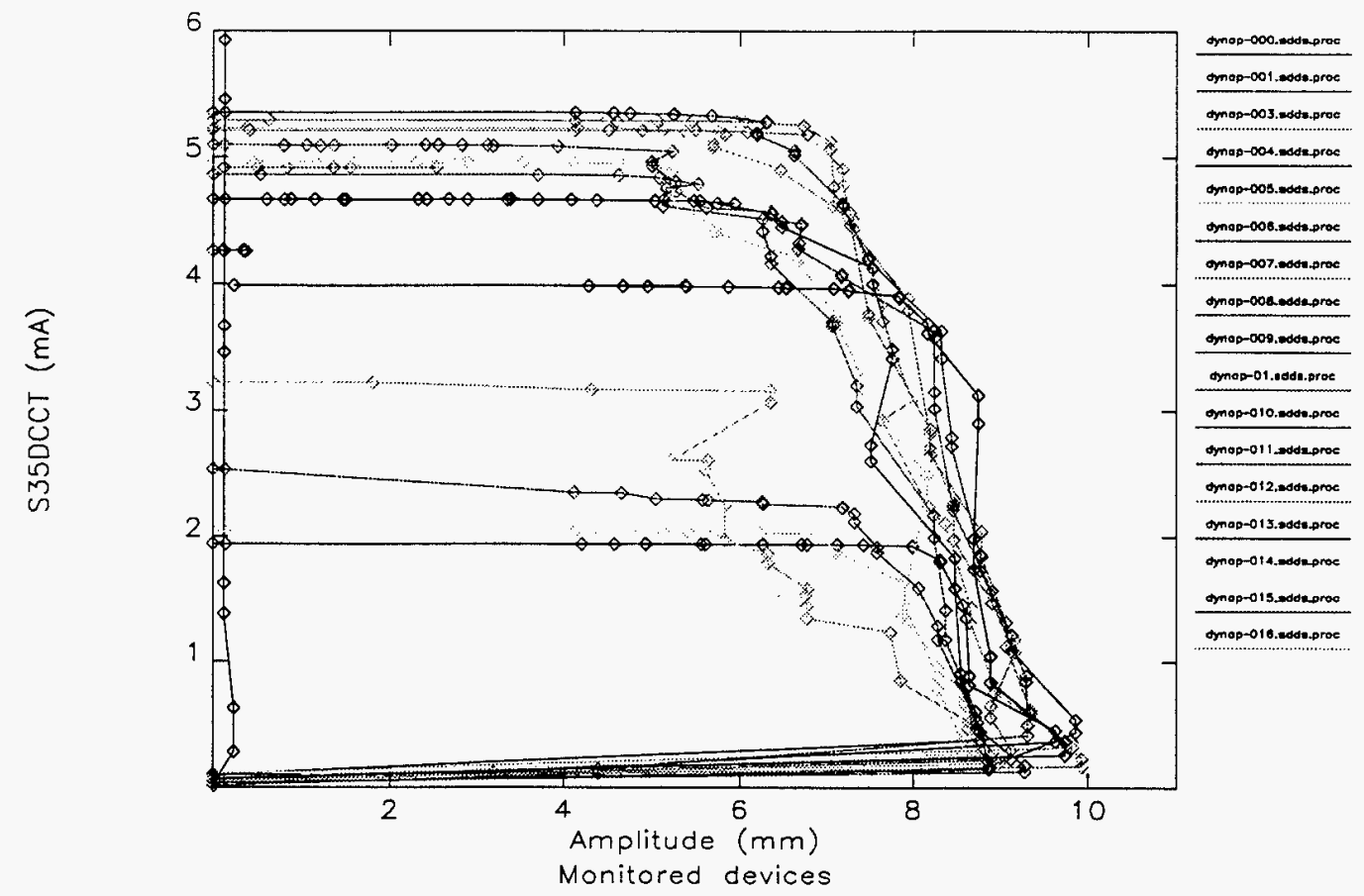

Figure 20. Intensity dependence on kicker-induced betatron amplitude

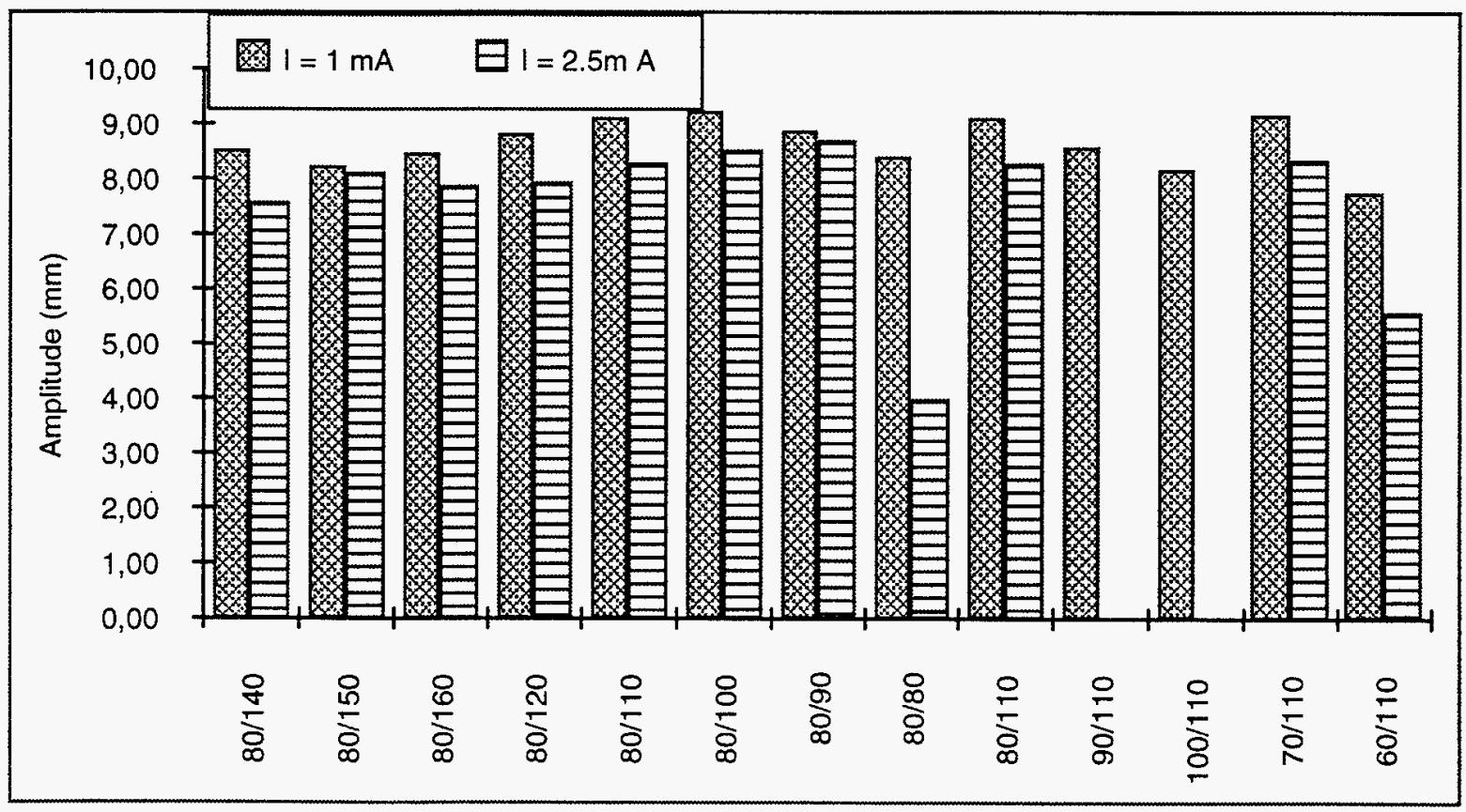

Figure 21. Kicker-induced betatron amplitude at two intensities 


\section{Evolution of dynamic aperture with closed orbit}

Measurements of the intensity dependence on kicker-induced betatron amplitude were performed with the user's orbit and the centered orbit, in order to assess the assumption of a dynamic aperture reduction induced by closed-orbit distortions. As shown in Figure 22, there are no significant differences between the two curves.

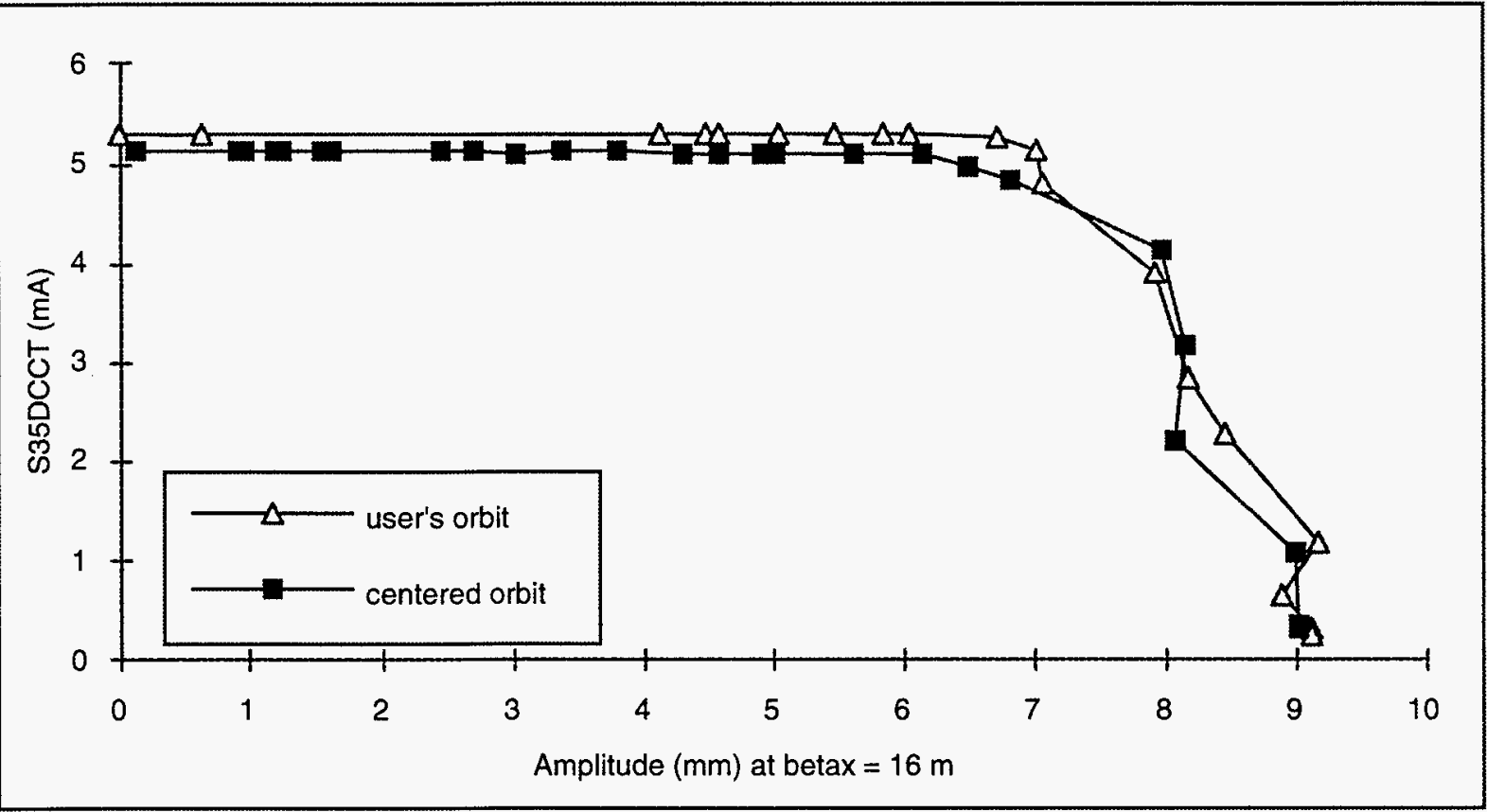

Figure 22. Comparison of intensity and kicker-induced betatron amplitude dependence on orbit

\section{Effect of scrapers}

The possibility of a horizontal dynamic aperture reduction induced by a strong horizontalvertical coupling was checked by performing a set of dynamic aperture measurements while progressively closing the S38 top scraper. Results in Figure 23 clearly indicate a nonlinear behavior of the vertical aperture and horizontal oscillation.

Since the calibration of the scraper position with respect to beam axis is not well known, it is assumed that the 2.4-cm position (last value before the horizontal amplitude starts to decrease) gives a physical aperture limitation equivalent to that of the 4-mm-high ID vessel. This enables the previous plot to be interpreted in terms of vertical limiting amplitude with respect to beam axis at the entrance of the ID vessel $\left(\beta_{\mathrm{y}}=6.76 \mathrm{~m}\right.$, as compared to $\beta_{\mathrm{y}} \sim 19 \mathrm{~m}$ at the scraper location). The nonlinear coupling at large amplitude clearly shows up (Figure 24). Determination of whether this horizontal-vertical coupling is induced by harmonic sextupole settings or by lattice errors requires additional experiments. In particular, it is not evident that 
two harmonic sextupole families are enough to minimize this detrimental effect. One could endeavor to define a 2-cell superperiodicity for the sextupoles and to move to four families.

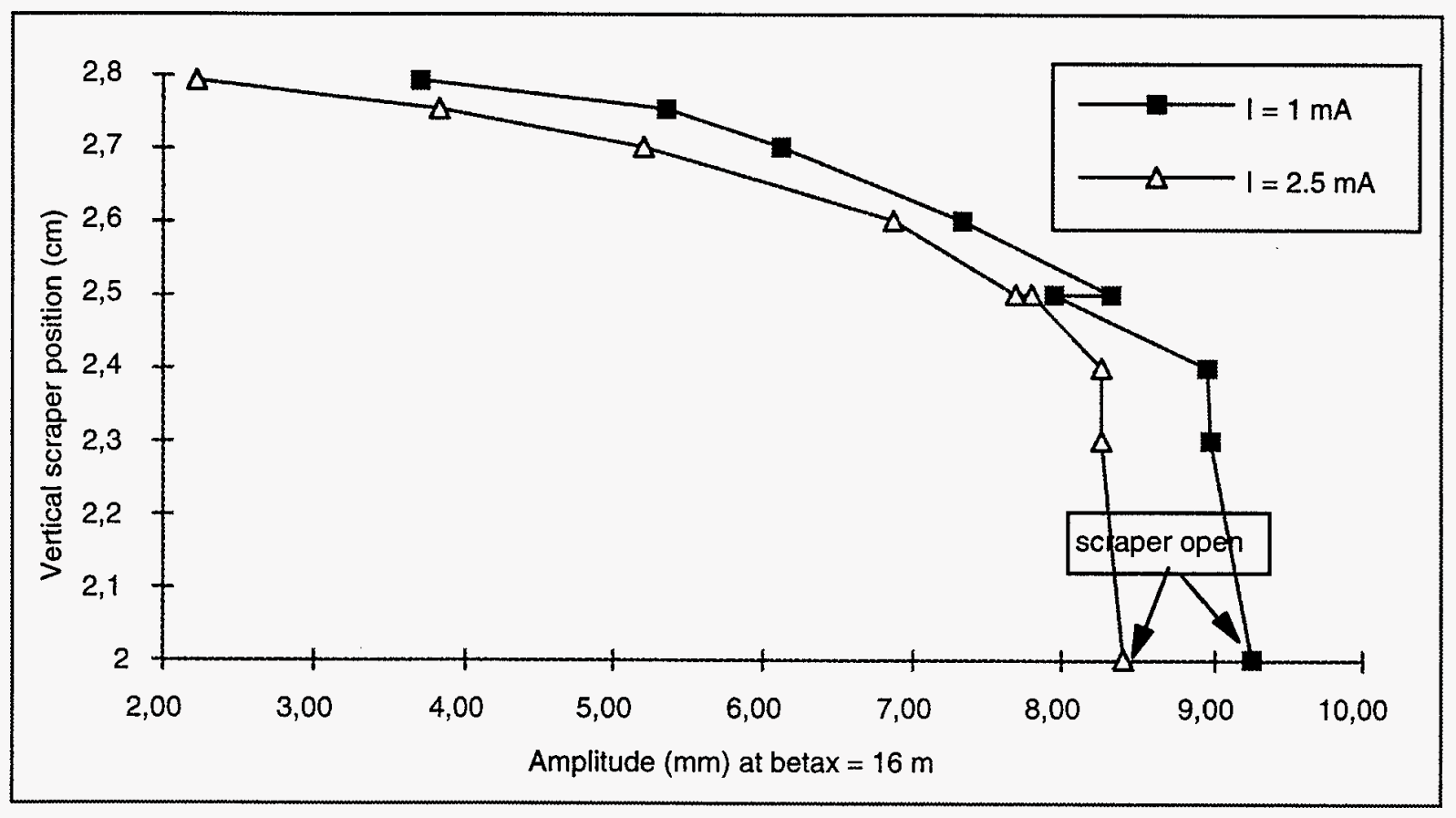

Figure 23. Dependence of maximum horizontal amplitude on vertical scraper closing

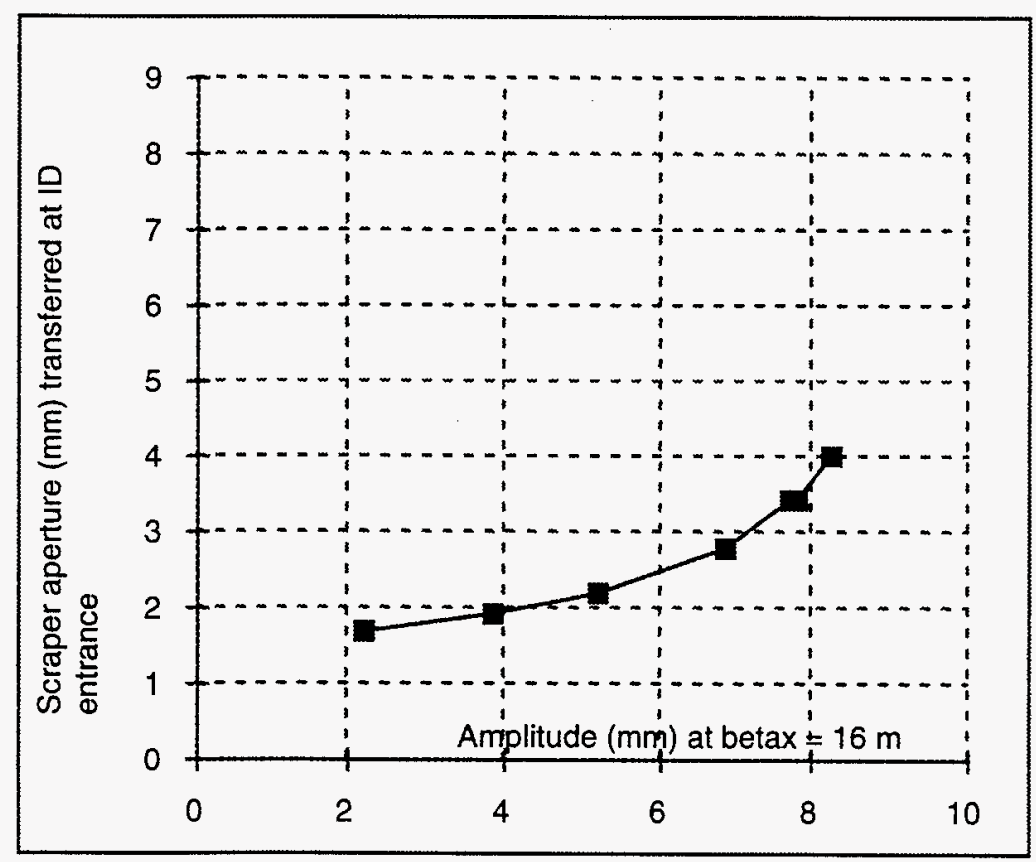

Figure 24. Linear and nonlinear horizontal/vertical coupling 
On the other hand, there is a nice linear dependence of the amplitude on the horizontal scraper position (Figure 25). A calibration of the scraper position would allow a cross-checking of the amplitude deduced from the BPM readings.

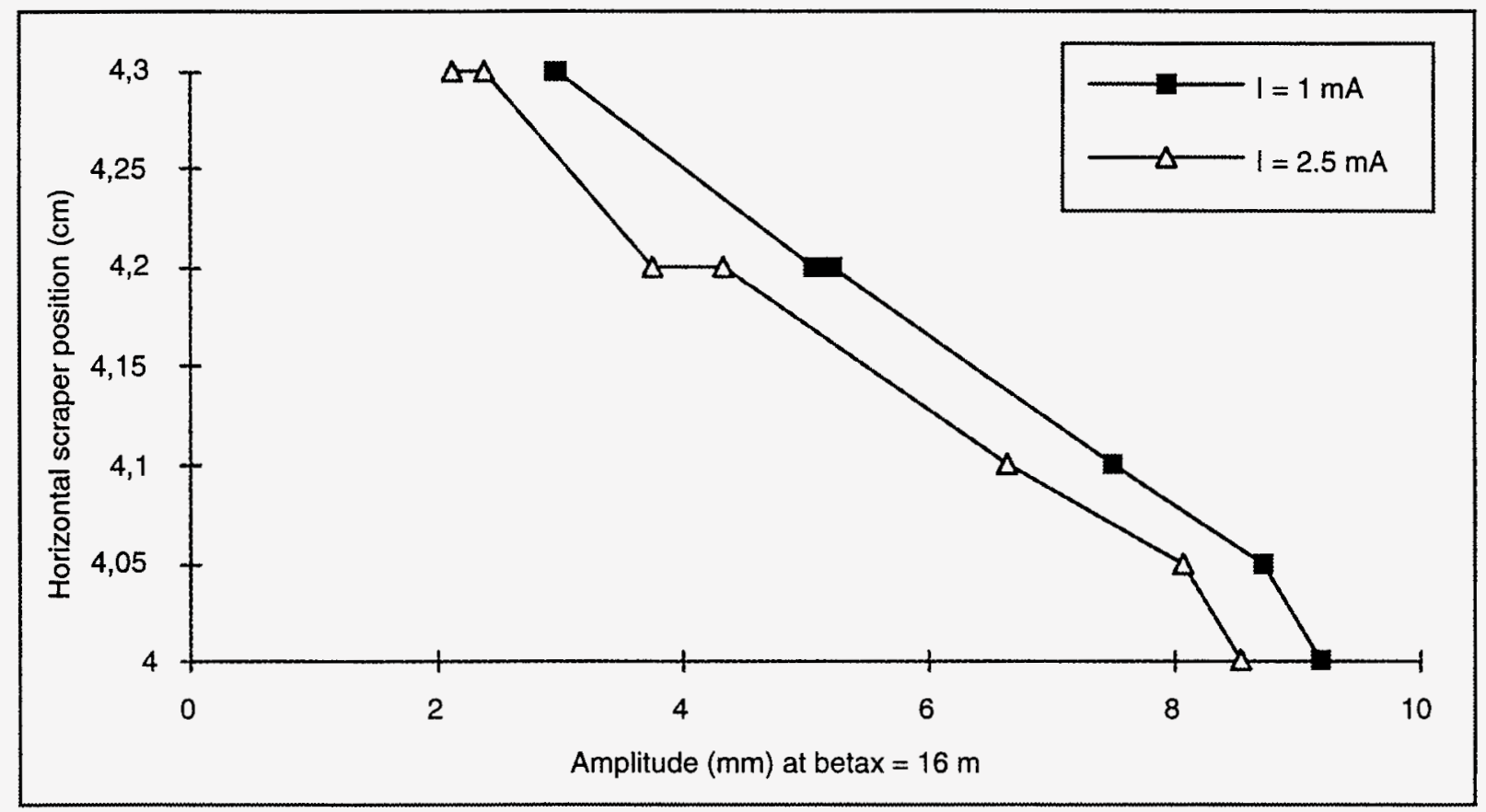

Figure 25. Dependence of maximum horizontal amplitude on horizontal scraper closing

\section{Closed-orbit-related characteristics}

As discussed in the first part of this Note, closed-orbit distortions might affect lattice characteristics. During the different machine studies, the following observations were recorded:

\section{1. $\underline{\text { Tunes }}$}

Tune change after a closed-orbit correction from $0.204 / 0.294\left(v_{\mathrm{x}} / v_{\mathrm{y}}\right)$ to $0.21 / 0.32$. This is obviously linked to the additional focusing from the off-centered beam in the sextupoles and has to be taken into account in the modeling of the machine.

\section{Dispersion}

Although the 5-fold periodicity remains, the dependence of the horizontal dispersion on the closed orbit clearly appears, with the modulation decreasing when the closed orbit is corrected at best (see Table 2 and Figures 26 and 27). Measurements with the centered orbit that had been performed at a 1-week interval and with a different correction algorithm are very reproducible. The distorted pattern could be due to the residual orbit $(0.25 \mathrm{~mm})$ or to the other sources of 
errors as mentioned earlier. It should also be pointed out that the vertical dispersion (one source of which is the vertical closed orbit in sextupoles) is also reduced when the orbit is centered. Although the vertical dispersion is most likely not the major contribution to the vertical emittance, its reduction is nevertheless valuable.

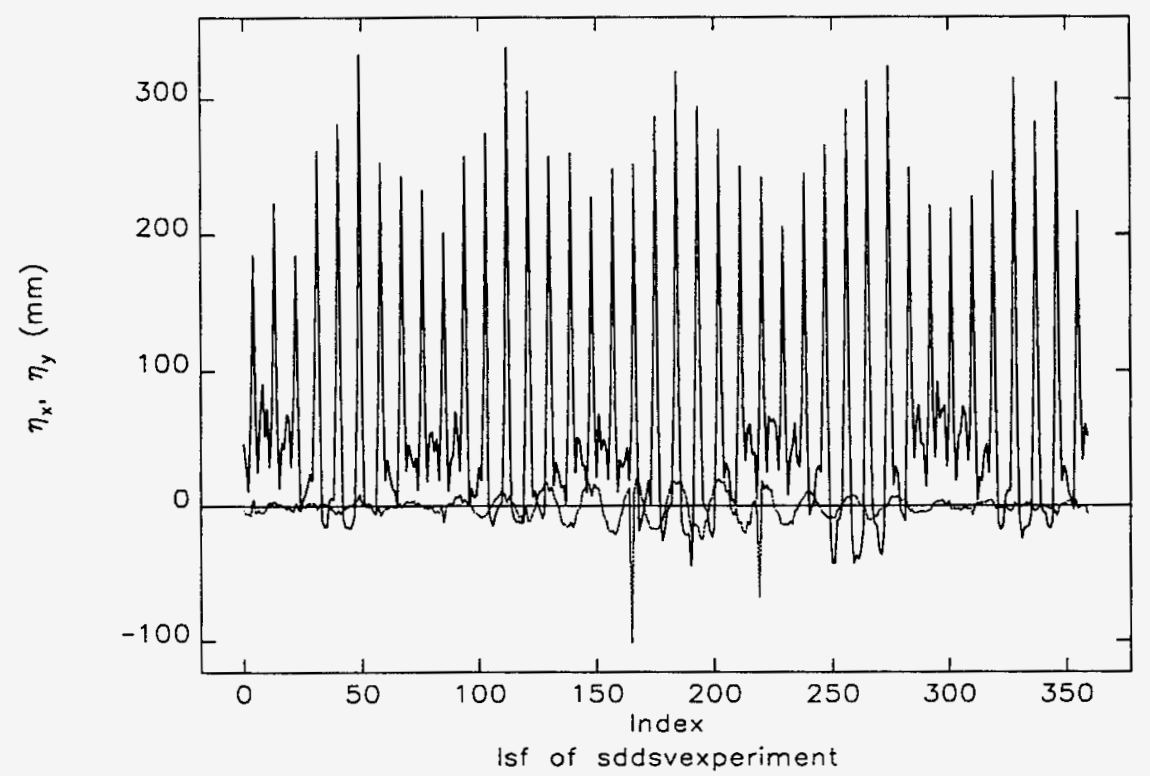

Figure 26. Measured dispersion with a partially centered orbit

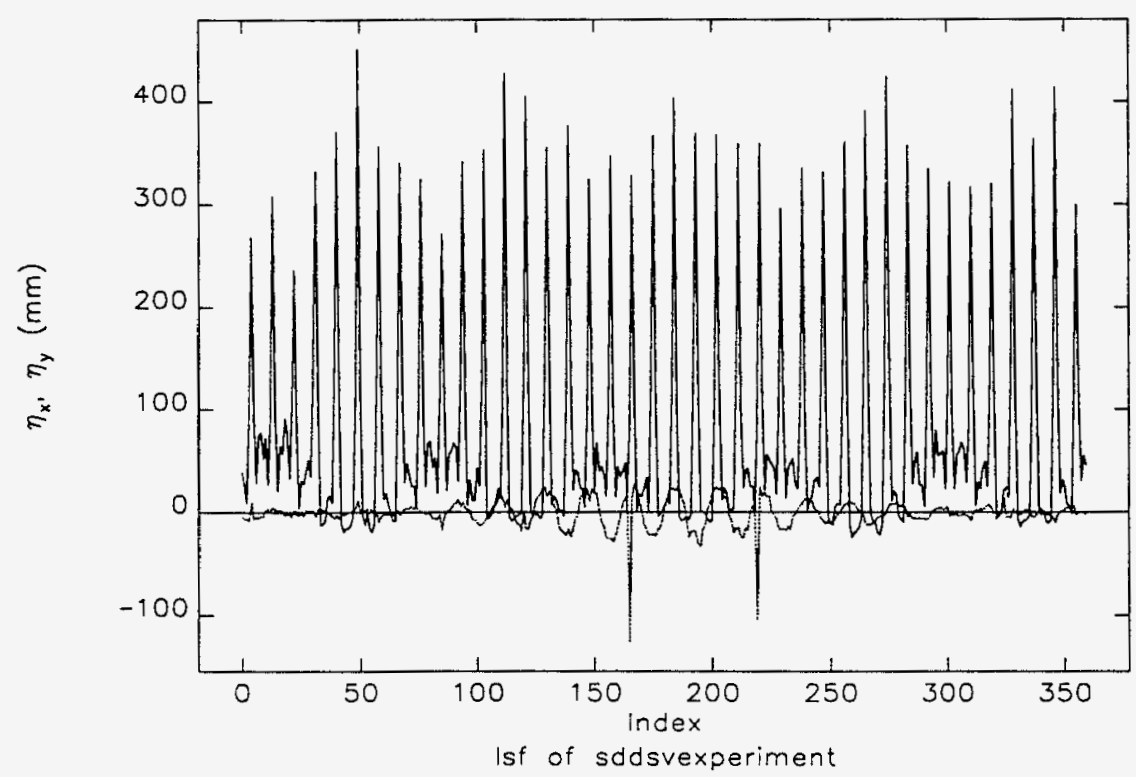

Figure 27. Measured dispersion with a centered orbit 
Table 2

\begin{tabular}{l|c|c|c} 
& partially centered orbit & centered orbit (10/28_1) & centered orbit (11/03_3) \\
\hline average $\eta_{\mathrm{x}}(\mathrm{mm})$ & 261.3 & 351 & 354 \\
\hline stdev $(\mathrm{mm})$ & 40 & 45 & 47 \\
\hline $\min \eta_{\mathrm{x}}(\mathrm{mm})$ & 183 & 236 & 239 \\
\hline $\max \eta_{\mathrm{x}}(\mathrm{mm})$ & 339 & 451 & 456 \\
\hline mod_min $/ \eta_{\text {theor }}$ & $-54 \%$ & $-41 \%$ & $-40 \%$ \\
\hline mod_max $/ \eta_{\text {theor }}$ & $-15 \%$ & $13 \%$ & $14 \%$
\end{tabular}

\section{Emittances and lifetime}

Monitoring the lifetime during the correction of the orbit at best with a $10 \mathrm{~mA} / 6$ bunches beam indicated a lifetime drop (see Figure 28); this could be the sign of smaller beam emittances.
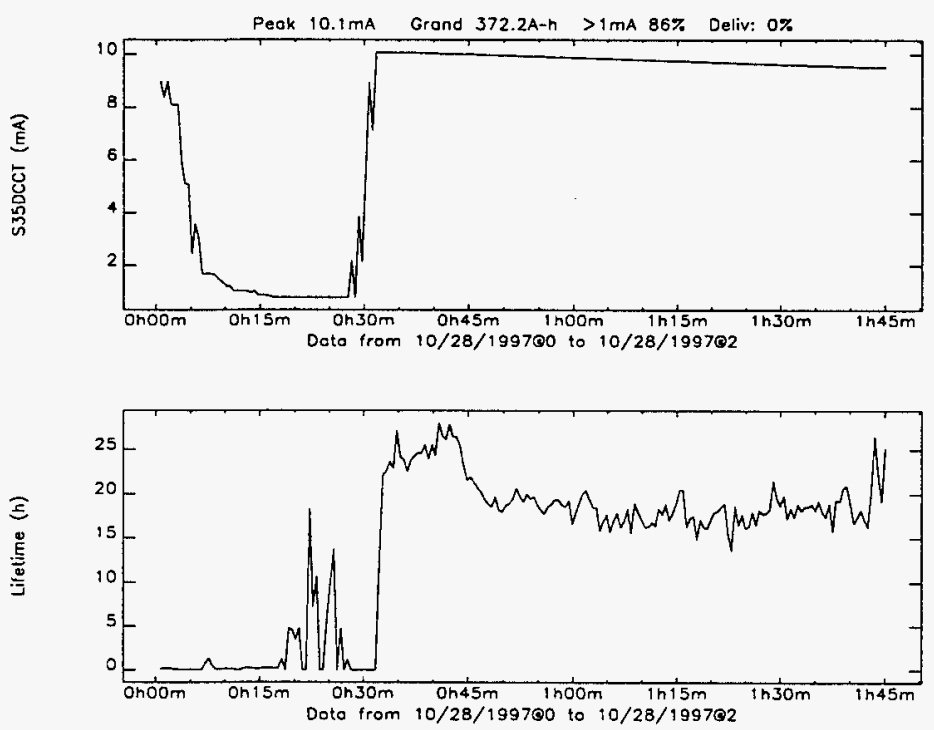

Figure 28. Evolution of lifetime with closed-orbit correction

This was qualitatively confirmed by observing the synchrotron light port at an intensity of 56 $\mathrm{mA}$ and measuring the raw beam sizes and the lifetime when switching from the user's orbit to the orbit corrected at best. As shown in Figure 29, the lifetime dropped from $43 \mathrm{~h}$ down to $35 \mathrm{~h}$. Simultaneously, FWHM beam sizes decreased from 40 to 30 pixels (horizontal) and from 22 to 17 pixels (vertical) with a brighter spot. Of course more systematic measurements are needed to quantify the impact of closed-orbit correction on emittance values. In particular, knowledge of lattice functions at the source point is absolutely necessary to avoid a mis-processing of the beam sizes. 

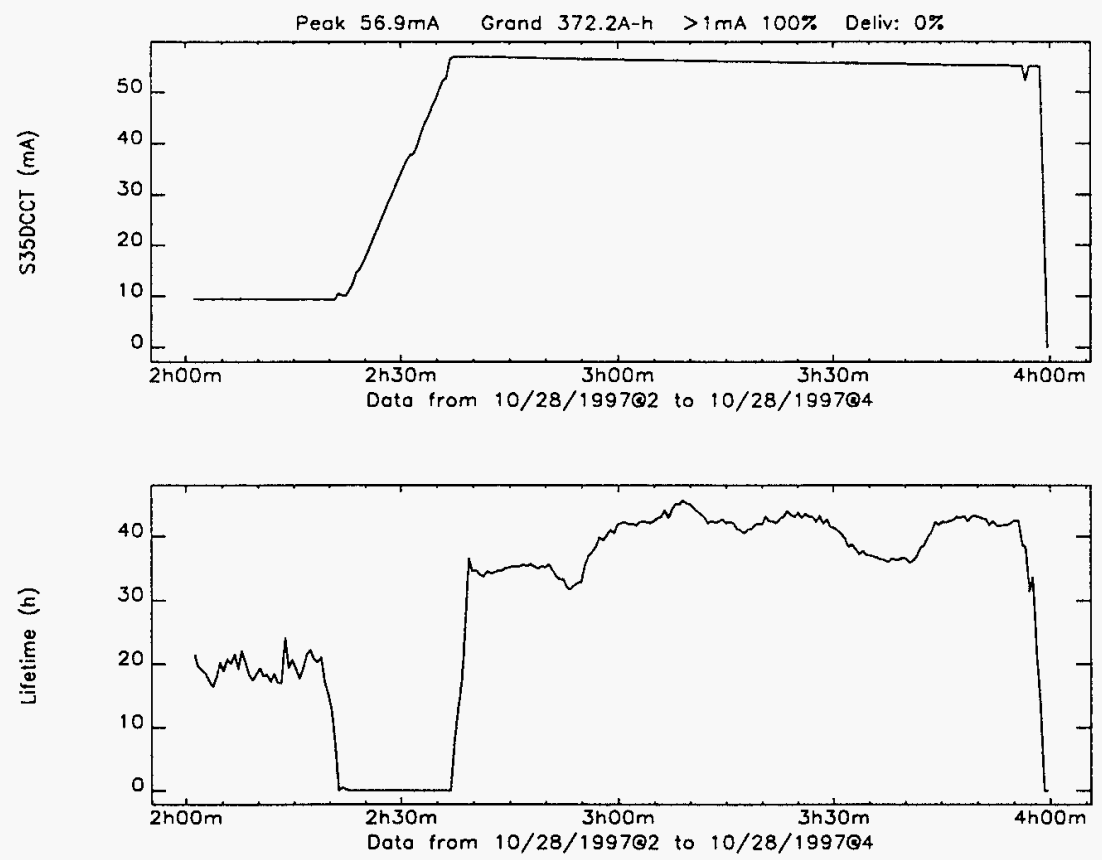

Figure 29. Lifetime with the user's orbit and with the centered orbit

\section{Effect of a local bump}

The simulation of the effects of a local bump on the lattice shows a significant deterioration of lattice characteristics with respect to the perfect machine: reduction of the dynamic aperture (Figure 30), modulation of $\beta$-functions (Figure 31) and large horizontal emittance blow-up of the horizontal dispersion (Figure 32). Even if the 40 -fold symmetry of the perfect lattice is already broken by the errors present in the real machine, a local bump should bring an additional perturbation, thus affecting machine performance.

Experimental testing was performed on the low beta-y insertion lattice by scanning a bump of increasing amplitude (set at the P2's BPM) in the low beta-y straight section (ID3) and recording the lifetime at $100 \mathrm{~mA}$ (Figure 33). As predicted, tune changes induced by the off-centered path of the particles in the sextupoles enclosed in the bump were almost negligible. Lifetime started to drop when the bump was set at $\pm 1.5 \mathrm{~mm}$. A reduction in lifetime by about a factor of 2 was experienced for the $+2 \mathrm{~mm}$ bump. Setting larger bumps induced a 40 -mA beam loss, very likely due to the 4-A delta limit used. Other features like beam emittances or $\beta$-functions were not measured. This would be useful to better quantify the effects of a bump. 


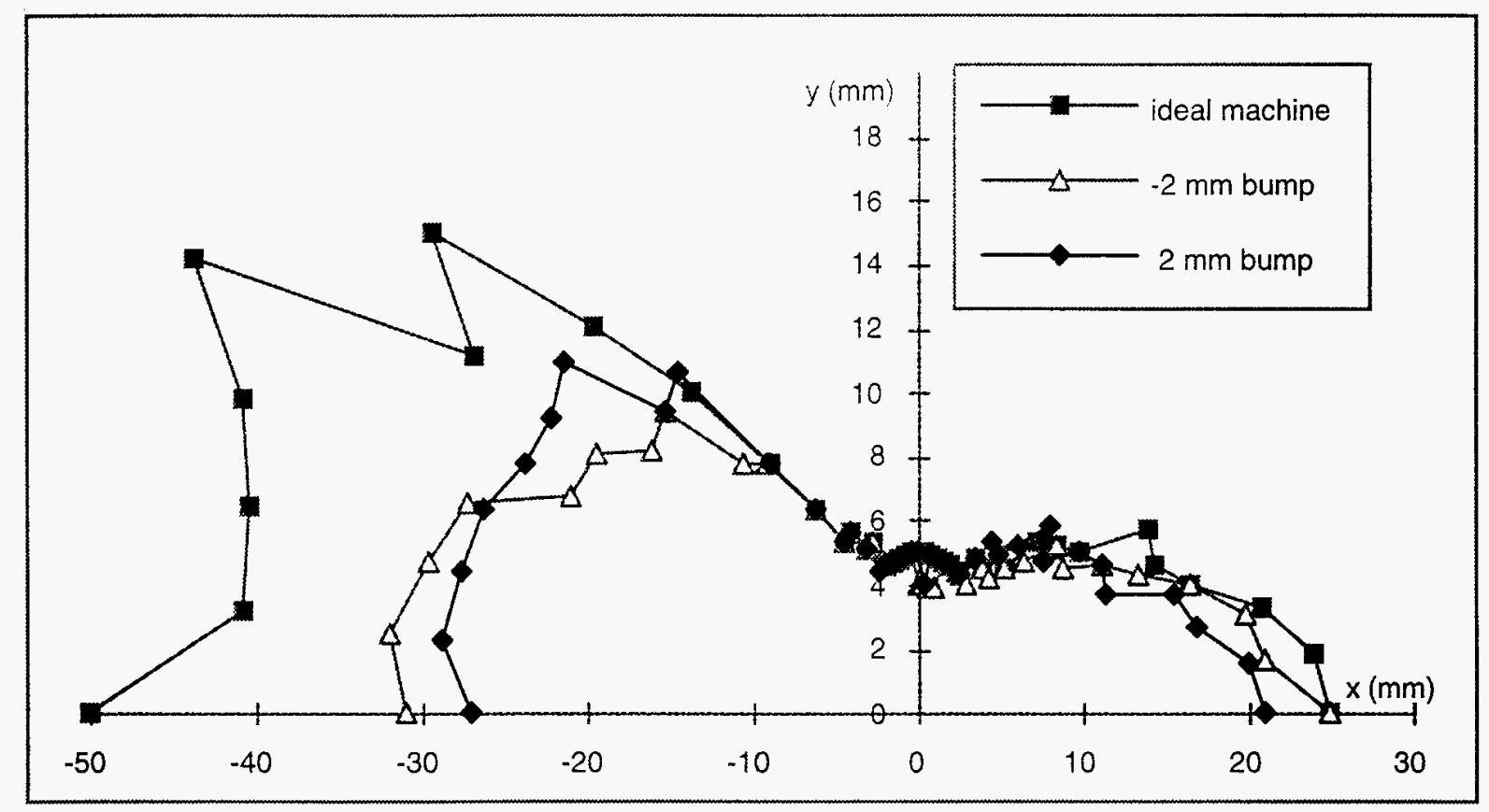

Figure 30. Dynamic aperture reduction induced by a local bump

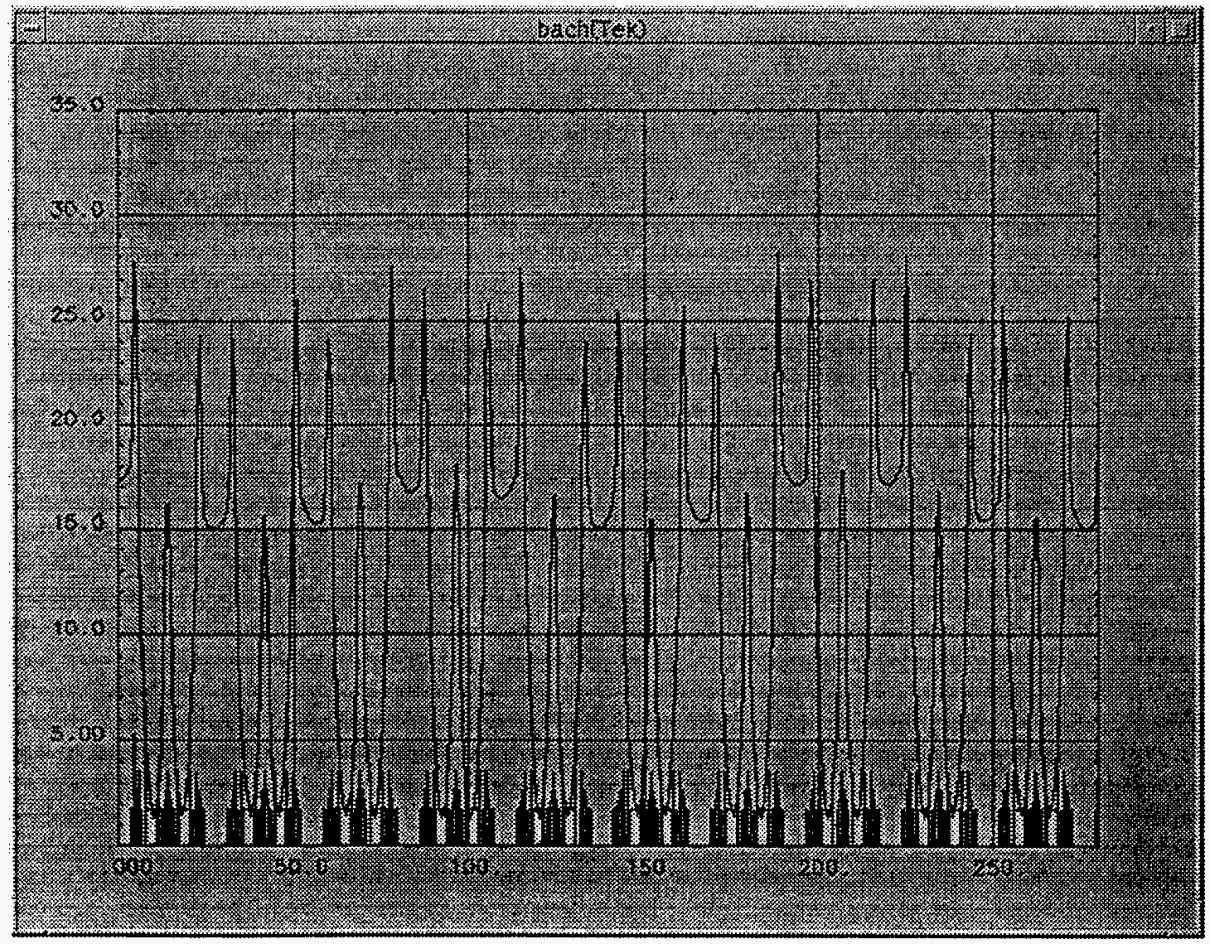

Figure 31. Horizontal $\beta$-function beat caused by a $-2 \mathrm{~mm}$ local bump 


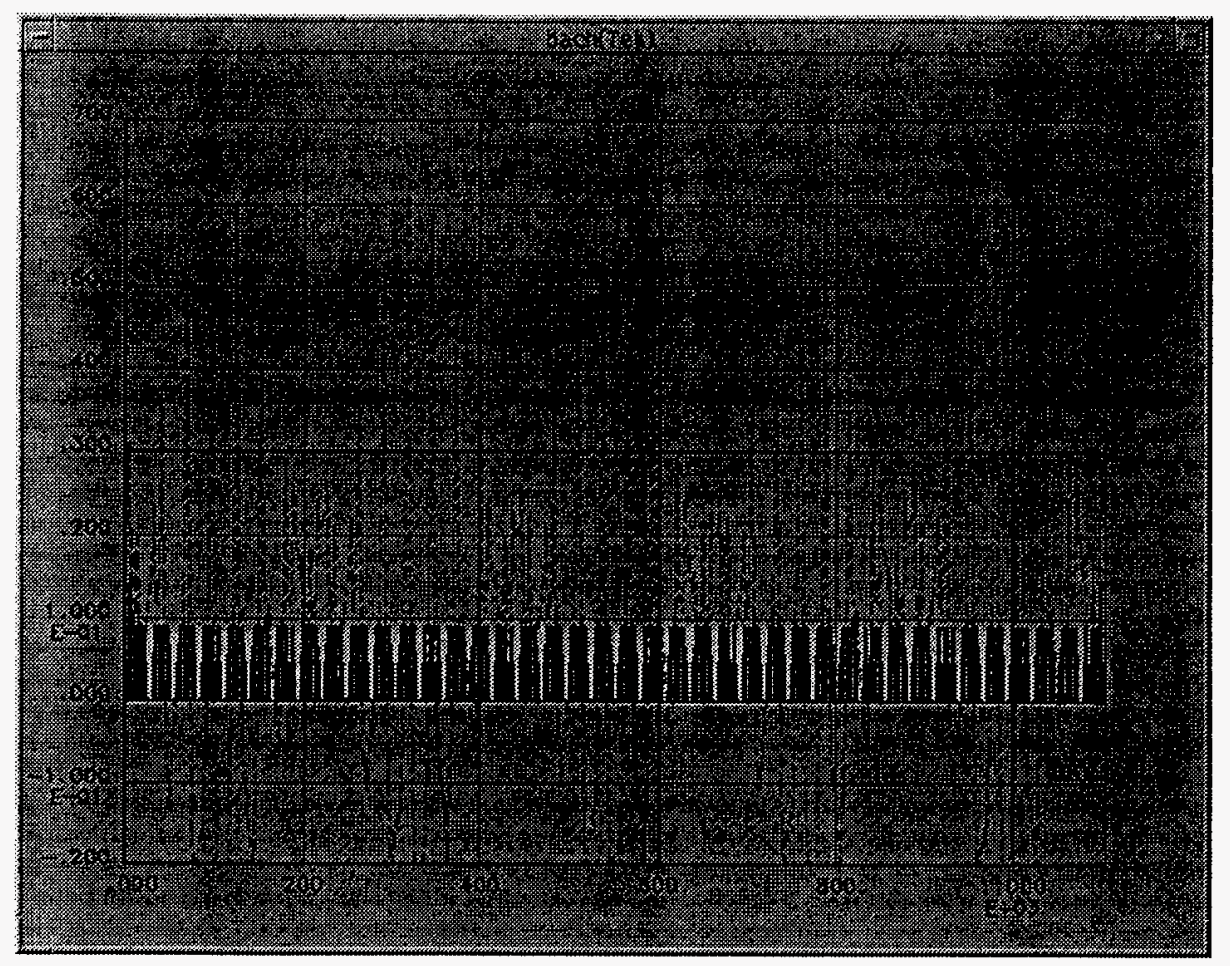

Figure 32. Horizontal dispersion modulation caused by a $-2 \mathrm{~mm}$ local bump
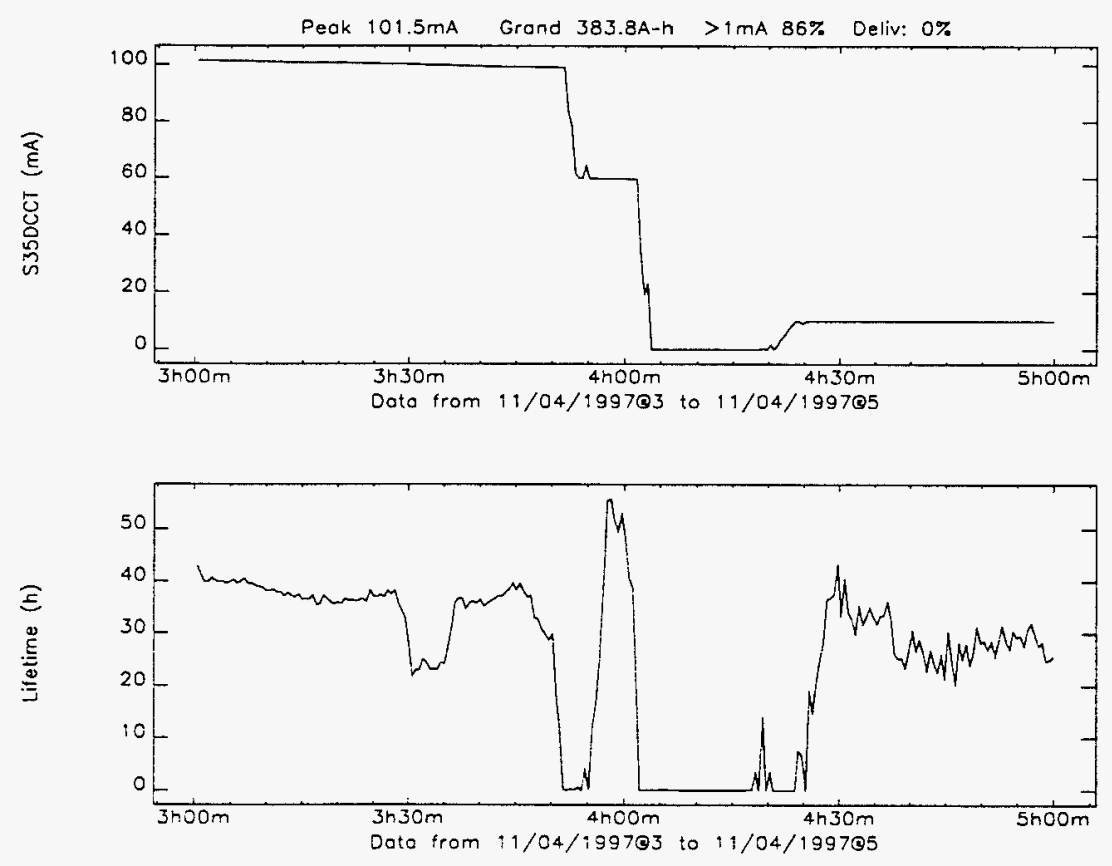

Figure 33. Effect of a local bump on the lifetime 


\section{Energy acceptance dependence on tunes}

Since the energy acceptance is determined by transverse limitations and not by $\mathrm{rf}$ limitations, the idea was to scan the working point in the tune diagram and to deduce the evolution of the energy acceptance from the $\mathrm{rf}$ frequency scan measurements. The experiment was performed at $\mathrm{I}=10$ $\mathrm{mA}$ in six bunches with the user's orbit for three different tunes (Table 3).

\section{Table 3}

\begin{tabular}{l|c|c} 
& $\begin{array}{c}\text { measured tunes } \\
v_{x} / v_{y}\end{array}$ & $\begin{array}{c}\text { measured chromaticities } \\
\xi_{\mathrm{x}} / \xi_{\mathrm{y}}\end{array}$ \\
\hline$v_{\mathrm{x}}=35.2, v_{\mathrm{y}}=19.3$ & $0.2110 / 0.3060$ & $0.31 \pm 0.26 / 4.24 . \pm 0.18$ \\
\hline$v_{\mathrm{x}}=35.25, v_{\mathrm{y}}=19.3$ & $0.2498 / 0.3141$ & $0.22 \pm 0.20 / 4.25 . \pm 0.18$ \\
\hline$v_{\mathrm{x}}=35.2, v_{\mathrm{y}}=19.25$ & $0.2055 / 0.2528$ & $0.92 \pm 0.15 / 3.54 . \pm 0.20$
\end{tabular}

The significant change in chromaticities for $v_{\mathrm{y}}=0.25$ looks surprising. Expected values are: $\xi_{\mathrm{x}}$ $=0.33, \xi_{y}=3.96$ ). For the three tunes, similar dispersion patterns with the same 5 -fold modulation were measured. Injection was possible for the three tunings (with the need, however, to lower the $\mathrm{K} 4$ kicker for $v_{\mathrm{y}}=0.25$, due very likely to an incorrect bump closure and to an insufficient vertical chromaticity).

The results of the energy acceptance measurements are summarized in Table 4 and in Figure 34.

Table 4

\begin{tabular}{l|c} 
& $\begin{array}{c}\Delta \mathrm{p} / \mathrm{p} \text { in \% } \\
\text { (defined at zero-lifetime) }\end{array}$ \\
\hline$v_{\mathrm{x}}=35.2, v_{\mathrm{y}}=19.3$ & $-1.85 / 1.31$ \\
\hline$v_{\mathrm{x}}=35.25, v_{\mathrm{y}}=19.3$ & $-1.68 / 0.81$ \\
\hline$v_{\mathrm{x}}=35.2, v_{\mathrm{y}}=19.25$ & $-1.81 / 1.56$
\end{tabular}




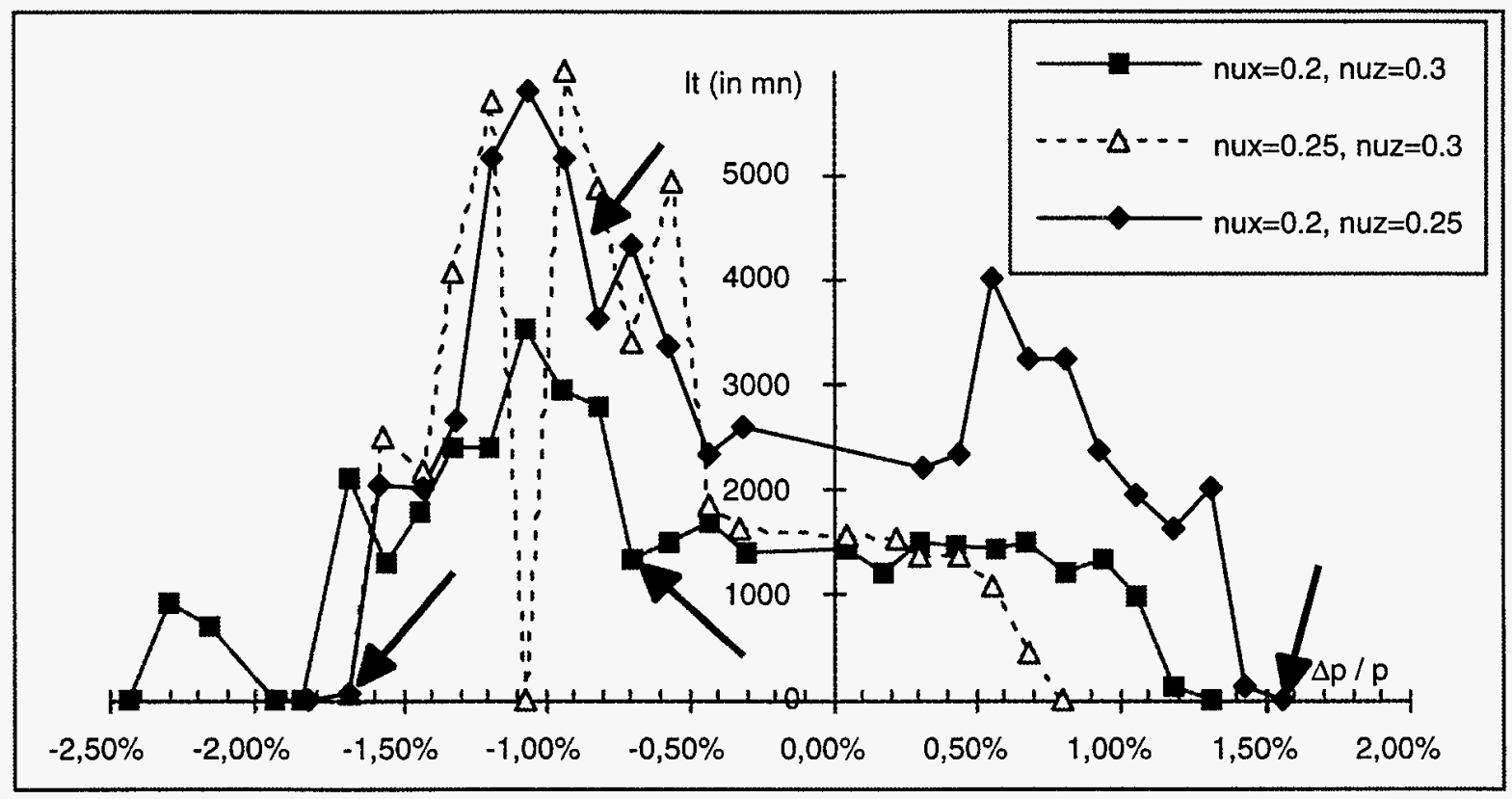

Figure 34. Energy acceptance dependence on tunes

All three curves show a dissymmetrical behavior for positive and negative energy deviations with a minimum effect for the $v_{x}=35.2, v_{y}=19.25$ tuning. The large evolutions in lifetime (increase or sudden drop to zero) are very likely due to resonance crossing. It was expected that the $v_{x}=35.25, v_{y}=19.3$ tuning would move the off-momentum tune path away from the suspected $2 v_{x}-v_{y}=51$ resonance (see Figure 35). Results are disappointing, and the small acceptance in energy is not understood. For all tunings, the off-momentum particles cross or approach the systematic fifth-order resonance $4 v_{x}+v_{y}=160$. This proximity might induce lifetime accidents (as sketched by the arrows in Figure 34). If this resonance really plays a negative role on the energy acceptance, there is little flexibility for escaping it in the tune diagram zone above the linear coupling resonance. In addition, any increase in chromaticity will favor the overlap of the off-momentum particles' tune path and of the dangerous resonances. 


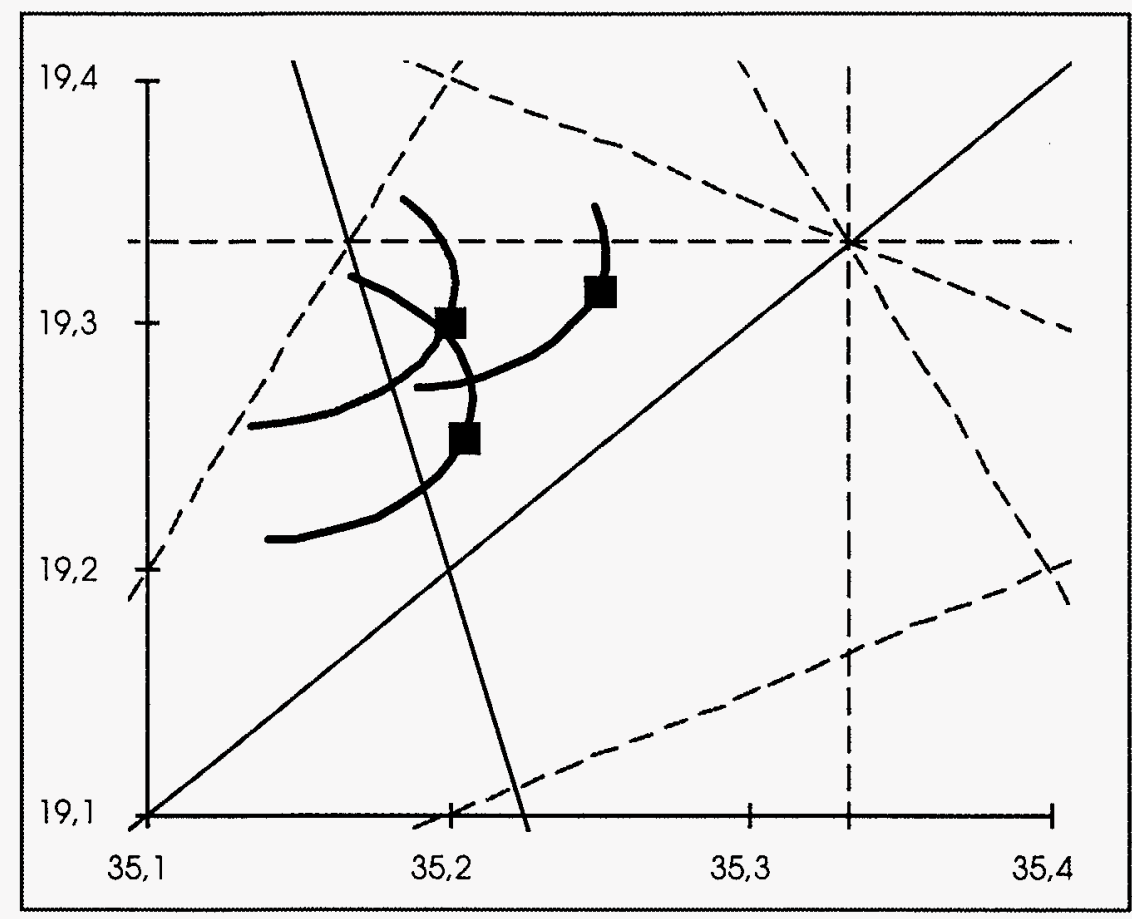

Figure 35. Tune path of off-momentum particles

\section{Low beta-y insertion}

Running the standard optics with a single low beta-y insertion is a good compromise for the installation of a 5-mm vacuum vessel whilst keeping the lattice functions unchanged in the rest of the ring. The strategy for matching the insertion to the regular cells is the following:

- Compute the vertical phase advance $\Delta \varphi_{y}$ for a $\beta_{y}=3 \mathrm{~m}$ straight section (the low beta-y insertion does not bring any change for the horizontal plane).

- Tune the nominal $v_{y}=14.3$ lattice to a vertical tune of $14.3-\Delta \varphi_{y}$ while keeping the horizontal tune at $v_{\mathrm{x}}=35.3$.

- Retune the triplet in one straight section in order to get a low beta-y insertion. If necessary, refine the tuning of the triplet of the normal cells.

The resulting quadrupole strengths (using quadrupole lengths from the magnetic measurements) are listed in Table 5. Lattice functions for 1/4 of the ring are plotted in Figure 36.

\section{$\underline{\text { Table } 5}$}

\begin{tabular}{l|c|c|c|c|c} 
& $\mathrm{Q} 1$ & $\mathrm{Q} 2$ & $\mathrm{Q} 3$ & $\mathrm{Q} 4$ & $\mathrm{Q} 5$ \\
\hline normal cells & -0.424077 & 0.649148 & -0.485058 & -0.788684 & 0.777522 \\
\hline low beta-y & -0.639673 & 0.785122 & -0.663141 & &
\end{tabular}




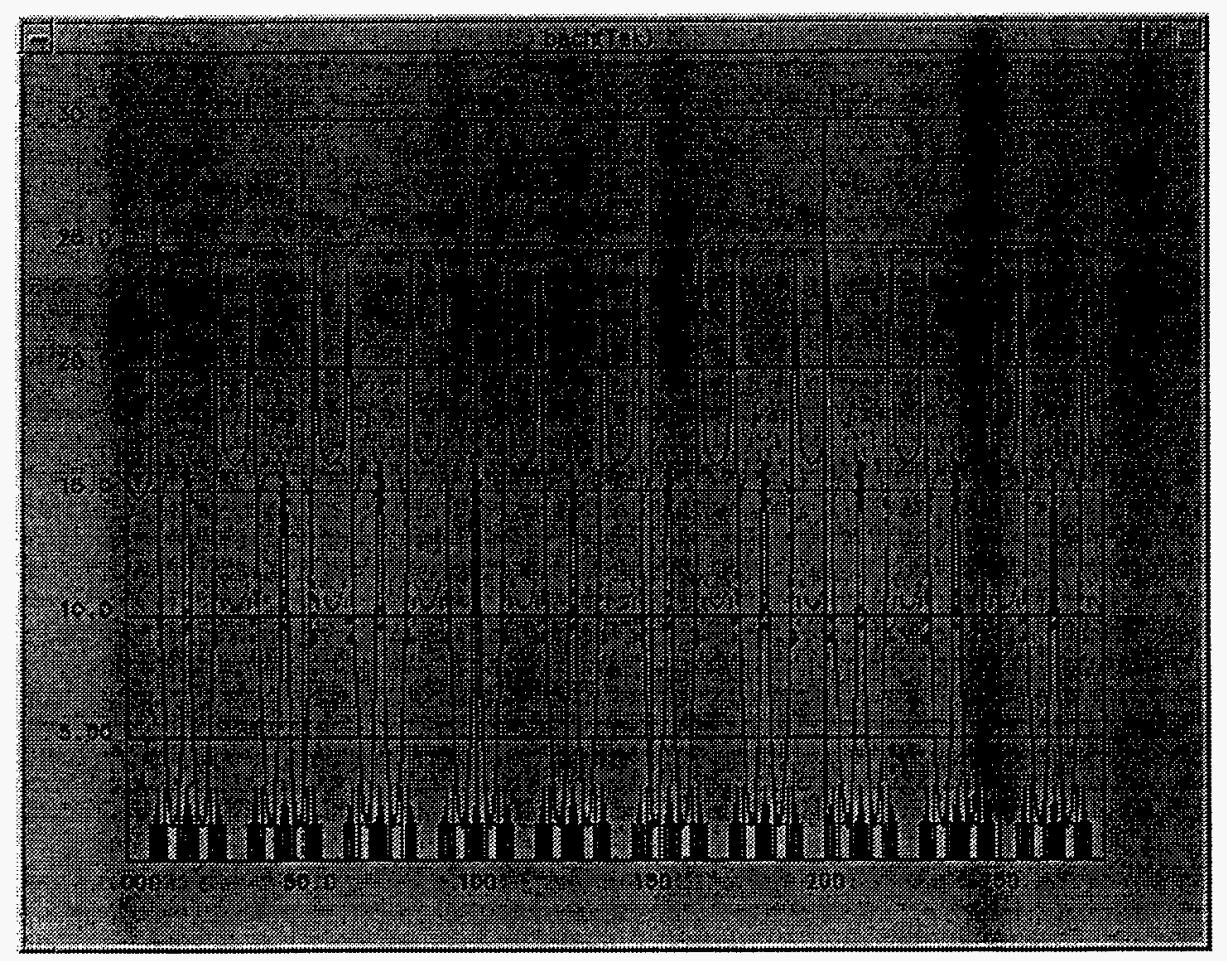

Figure 36. Lattice functions of the low beta-y insertion optics

Figure 37 gives a comparison of the dynamic apertures of the perfect machine for the three optics (standard $v_{y}=14.3$ optics, low beta-y optics tuned at $v_{y}=19.3$, and low beta-y insertion optics tuned at $v_{\mathrm{y}}=14.3$ ). Apertures are quoted in terms of number of standard deviations ( $N_{x, y}=\frac{x, y}{\sigma_{x, y}}$, with $\sigma_{y}$ defined for full coupling) in order to get comparable figures for optics with different $\beta$ s. Clearly the low beta-y insertion optics shows a larger vertical dynamic aperture than the low beta-y optics. It would be interesting to remeasure the evolution of horizontal aperture versus vertical scraper and compare the evolution of the coupling between horizontal and vertical planes.

The experimental difficulty for testing the low beta-y insertion optics is in keeping the theoretical matching, which could be difficult given the differences between the real machine and the model. The net consequences of a mismatch would be a $\beta$-function beat all along the machine as sketched in Figure 38. 


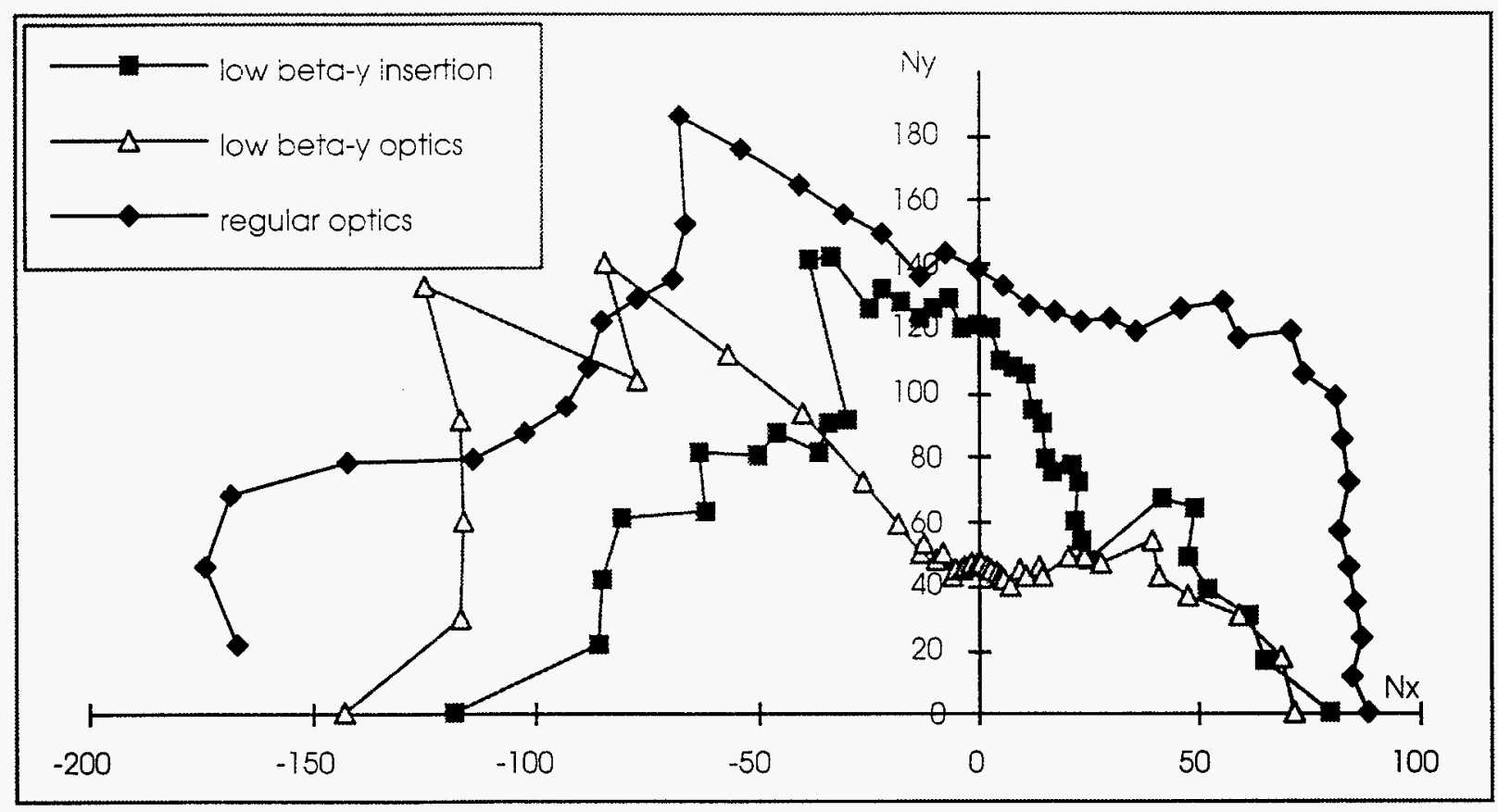

Figure 37. Comparison of dynamic apertures for the three optics

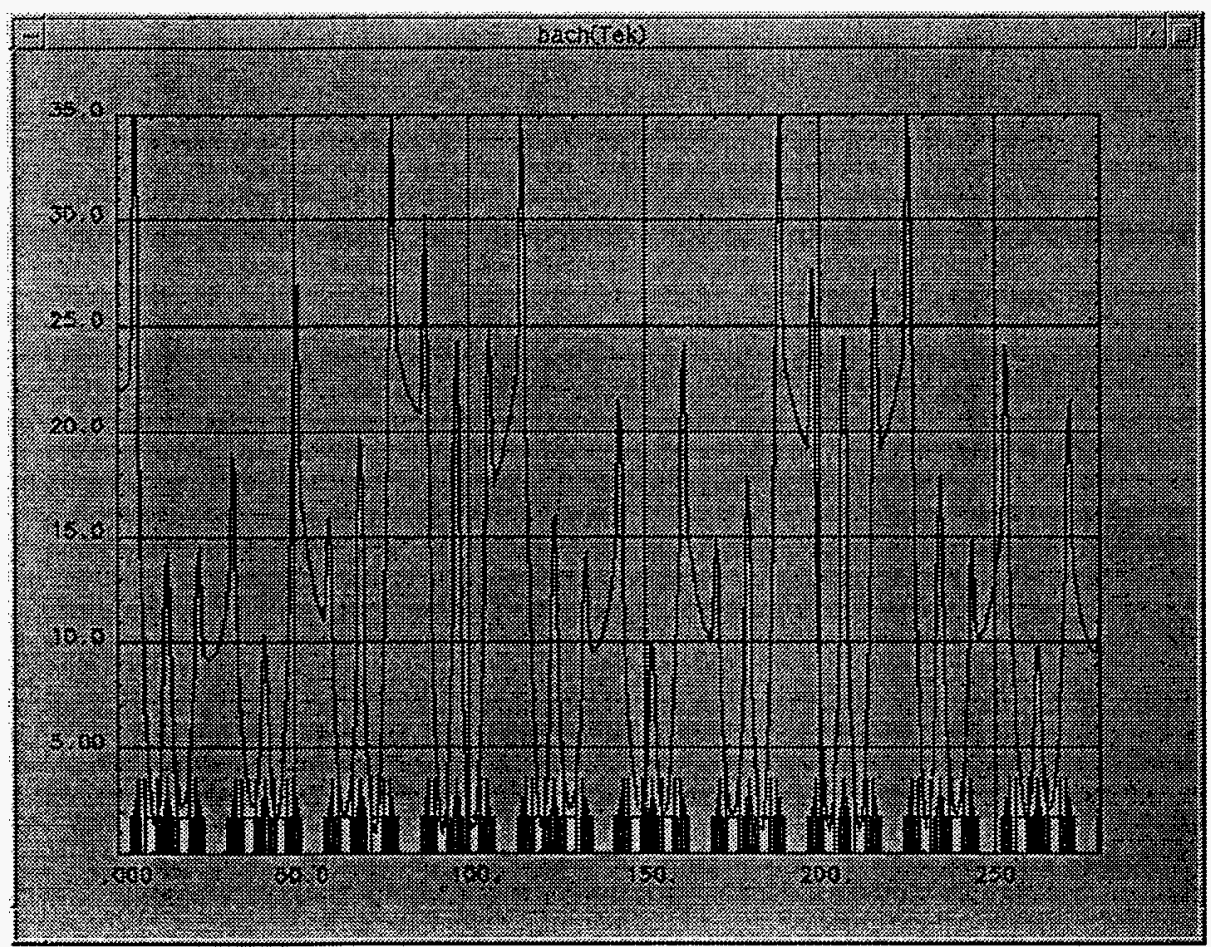

Figure 38. Horizontal $\beta$-function beat with a mismatched insertion (1/4 of the machine) 


\section{Conclusions}

The steering for users leads to a rather large orbit that induces a significant degradation of the lattice features in simulations. All the expected negative effects of the user's orbit could not be identified in an unambiguous way during the dedicated machine studies. However, part of the experimental distortions of the lattice characteristics (tunes, $\beta$-function beat, modulation of the horizontal dispersion) is likely induced by this large orbit. A good modeling of the optics certainly requires removal of these perturbations.

Although the two measuring techniques (rf frequency or voltage scan) do not fully agree on the limiting figures, the energy acceptance of the machine appears to be transversally limited and not rf limited. Possible candidates for explaining the limitation could be a random third-order resonance or a systematic fifth-order resonance on the path of Touschek-scattered particles.

There are clear indications that a corrected orbit leads to smaller emittances (which is a nice feature). The unpleasant consequence is a smaller Touschek lifetime. On-line emittance measurements would be helpful to get quantitative data.

Even if the measured 10-mm horizontal dynamic aperture is adequate to accommodate the induced betatron oscillations at injection, it is far below predicted figures. A large nonlinear horizontal-vertical coupling might be the cause of the discrepancy between simulations and measurements. In that respect, the low beta-y insertion optics looks much more forgiving than the low beta-y optics. This nonlinear horizontal-vertical coupling could be the dominant source of dynamic aperture reduction as compared to closed-orbit errors. Using only two harmonic sextupole families might be insufficient to minimize this effect.

\section{Acknowledgments}

I would like to thank particularly M. Borland, L. Emery, and N. Sereno for having eased my first contacts with the APS control system and the data processing, and for having conducted the machine studies that have been analyzed in this report. 


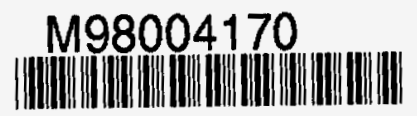

Report Number (14) LS $=-264($ ANL)

Publ. Date (11) 199803

Sponsor Code (18) DOE/ER, XF

UC Category (19) UC-400; DOE/ER 